\title{
Reaction-diffusion processes and their connection with integrable quantum spin chains $^{1}$
}

\author{
Malte Henkel \\ Laboratoire de Physique des Matériaux (CNRS UMR 7556) et \\ Laboratoire Européen de Recherche Universitaire Sarre-Lorraine, \\ Université Henri Poincaré Nancy I, B.P. 239, \\ F - 54506 Vandœuvre lès Nancy Cedex, France
}

\begin{abstract}
This is a pedagogical account on reaction-diffusion systems and their relationship with integrable quantum spin chains.

Reaction-diffusion systems are paradigmatic examples of non-equilibrium systems. Their long-time behaviour is strongly influenced through fluctuation effects in low dimensions which renders the habitual mean-field kinetic equations inapplicable. Starting from the master equation rewritten as a Schrödinger equation with imaginary time, the associated quantum Hamiltonian of certain one-dimensional reaction-diffusion models is closely related to integrable magnetic chains. The relationship with the Hecke algebra and its quotients allows to identify integrable reaction-diffusion models and, through the Baxterization procedure, relate them to the solutions of Yang-Baxter equations which can be solved via the Bethe ansatz. Methods such as spectral and partial integrability, free fermions, similarity transformations or diffusion algebras are reviewed, with several concrete examples treated explicitly. An outlook on how the recently-introduced concept of local scale invariance might become useful in the description of nonequilibrium ageing phenomena is presented, with particular emphasis on the kinetic Ising model with Glauber dynamics.
\end{abstract}

\footnotetext{
${ }^{1}$ to be published in: A. Kundu (éd) Classical and Quantum Nonlinear Integrable Systems: Theory and Applications, Institute of Physics Publishing (Bristol 2003)

ISBN 0750309598
} 


\section{Reaction-diffusion processes}

The understanding of non-equilibrium statistical physics is still much more incomplete than that of equilibrium theory, due to the absence of an analogue of the Boltzmann-Gibbs approach and in spite of considerable recent progress [1. Therefore, non-equilibrium systems have to be specified by some defining dynamical rules which are then analyzed. The topic has received a lot of attention and many reviews exist, e.g. 2, 3, 4, 5, 6, 7, 8, 9, 9. Exactly solvable systems far from equilibrium have been recently reviewed in a nice way [10. Here I present a pedagogically-minded introduction to the application of a few standard tools from one-dimensional integrable quantum systems to nonequilibrium statistical mechanics. After recalling why standard descriptions such a kinetic or reaction-diffusion differential equations are in general insufficient in one dimension, we remind the reader in section 2 on the quantum Hamiltonian formulation of non-equilibrium processes which in turn is based on the master equation. Section 3 recalls a few basic facts about Hecke algebras. These building blocks are used in sections 4 and 5 to show explicitly the integrability of certain single-species reaction-diffusion processes, through their relation to integrable vertex models. Section 6 reviews some further methods such as spectral and partial integrability, the free-fermion technique, similarity transformations or diffusion algebras. We close in section 7 with an outlook on how the recently-introduced concept of local scale invariance might become useful in the description of non-equilibrium ageing phenomena.

I have made no effort to provide a complete bibliography. This may be found in the excellent reviews quoted above.

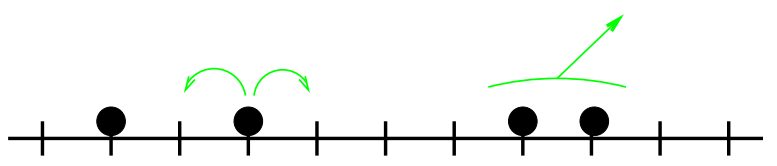

Figure 1: Microscopic reactions in the diffusive pair-annihilation process.

A class of non-equilibrium models which are particularly simple to formulate are the so-called reaction-diffusion processes. Consider the following example: particles of a single kind (species) move on a lattice (figure 1). Each site of the lattice may be either empty (denoted by o) or else be occupied by a single particle (denoted by $\bullet$ ). The particles are allowed to undergo the 
following movements, see figure 1, which involve the states of two nearestneighbour sites:

$$
\begin{array}{ll}
\circ+\bullet \leftrightarrow \bullet+\circ & \text { diffusion, with rate } D \\
\bullet+\bullet \rightarrow \circ+\circ & \text { pair-annihilation, with rate } 2 \alpha
\end{array}
$$

The first of the allowed movements in (11) is reversible while the second is not. A typical question is then for the long-time behaviour of quantities such as the mean particle density $n(t)$. Trivially $n(t)$ decreases with increasing time $t$ but different long-time asymptotic behaviours such as $n(t) \sim t^{-\alpha}$ or $n(t) \sim e^{-t / \tau}$ are conceivable. The oldest approach to this problem was introduced by Smoluchowski [1] and consists of writing down kinetic equations, e.g. for the spatially averaged density $n(t)$ and one obtains $\partial_{t} n(t)=-\lambda n(t)^{2}$ for the problem at hand, where $\lambda=4 \alpha$. With the initial condition $n(0)=n_{0}$, the solution $n(t)=n_{0}\left(1+n_{0} \lambda t\right)^{-1} \simeq(\lambda t)^{-1}$ is easily found and apparently answers the physical question. A slightly more involved version of this argument does allow for spatial variation of the density $n=n(\boldsymbol{r}, t)$ and considers a reaction-diffusion equation

$$
\partial_{t} n(\boldsymbol{r}, t)=D \nabla^{2} n(\boldsymbol{r}, t)-\lambda n(\boldsymbol{r}, t)^{2}
$$

While the analysis of such non-linear partial differential equations is a formidable problem in its own right, these equations do not yet capture the essential physics in low-spatial dimensions, as we now show. Rather, they must be considered as an approximation of mean-field type.

In order to understand the approximative nature of equations such as (2), and following [12], consider the mean particle-density in a large volume $V$

$$
\bar{n}(t)=\frac{1}{V} \int_{V} \mathrm{~d} \boldsymbol{r} n(\boldsymbol{r}, t)
$$

It then follows

$$
\begin{aligned}
\partial_{t} \bar{n}(t) & =\frac{1}{V} \int_{V} \mathrm{~d} \boldsymbol{r}\left[D \nabla^{2} n(\boldsymbol{r}, t)-\lambda n(\boldsymbol{r}, t)^{2}\right] \\
& =\frac{D}{V} \int_{\partial V} \mathrm{~d} \boldsymbol{\sigma} \cdot \boldsymbol{\nabla} n(\boldsymbol{r}, t)-\frac{\lambda}{V} \int_{V} \mathrm{~d} \boldsymbol{r} n(\boldsymbol{r}, t)^{2} \\
& \leq-\lambda\left(\frac{1}{V} \int_{V} \mathrm{~d} \boldsymbol{r} n(\boldsymbol{r}, t)\right)^{2}
\end{aligned}
$$


In the second line, Gauß' theorem was used where d $\boldsymbol{\sigma}$ is normal to the surface $\partial V$ (the flow through $\partial V$ vanishes for large volumes $V \rightarrow \infty$ ). The last line follows from the Cauchy-Schwarz inequality. Together with the initial condition $\bar{n}(0)=n_{0}$, the inequality $\partial_{t} \bar{n}(t) \leq-\lambda \bar{n}(t)^{2}$ yields the bound

$$
\bar{n}(t) \leq \frac{n_{0}}{1+n_{0} \lambda t} \leq \frac{1}{\lambda t}
$$

for all times $t \geq 0$. However, the model defined above can be solved exactly in one spatial dimension (in a setting defined precisely in section 3), provided $D=2 \alpha$. The exact mean particle-density is given by [13]

$$
\begin{aligned}
\bar{n}(t) & =n_{0} e^{-4 D t}\left[I_{0}(4 D t)+2\left(1-n_{0}\right) \sum_{k=1}^{\infty}\left(1-2 n_{0}\right)^{k-1} I_{k}(4 D t)\right] \\
& \simeq \frac{1}{\sqrt{8 \pi D}} t^{-1 / 2} ; t \rightarrow \infty
\end{aligned}
$$

where $I_{k}$ is the modified Bessel function of order $k$. Clearly, for large times the exact mean density $\bar{n}(t)$ decreases considerably slower than even the upper bound (5) derived from the reaction-diffusion equation (2).

The failure of eq. (2) to describe correctly the long-time behaviour can be understood from the following heuristic argument [14]. In the long-time limit, the particle-density should already be very low and it is conceivable that at most one annihilation reaction takes place at a given time. Let $L=L(t)$ be the typical distance between two particles. Then the time needed by diffusive motion to overcome this distance is of the order $t \sim L(t)^{2}$. On the other hand, the mean particle density is $\bar{n}(t) \sim L(t)^{-d}$ in $d$ spatial dimensions and this argument would give $\bar{n}(t) \sim t^{-d / 2}$. Therefore, the assumption implicit in equations such as (2) that diffusive motion can render the system sufficiently homogeneous fails in low dimensions (in our model for $d<2$ ) and one rather has the long-time behaviour 14

$$
\bar{n}(t) \sim \begin{cases}t^{-d / 2} & \text { if } d<2 \\ t^{-1} & \text { if } d>2\end{cases}
$$

Therefore $d^{*}=2$ is the upper critical dimension of the diffusive pair-annihilation process. For dimensions $d>d^{*}$, reaction-diffusion equations should be expected to give qualitatively correct results and entire branches of physical chemistry are built on this. On the other hand, for low-dimensional structures 
Table 1: Measured decay exponent $y$ of the mean exciton density $\bar{n}(t) \sim t^{-y}$ on polymer chains. The error bar for TMMC comes from averaging over the results of [17] for different initial particle densities.

\begin{tabular}{|c|c|c|c|c|}
\hline substance & $y$ & \multicolumn{2}{|c|}{ reaction(s) } & Reference \\
\hline $\mathrm{C}_{10} \mathrm{H}_{8}$ & $0.52-0.59$ & $\bullet \bullet \rightarrow$ & $\begin{array}{l}\circ 0 \\
\bullet ०\end{array}$ & 15 \\
\hline P1VN/PMMA film & $0.47(3)$ & $\bullet \bullet \rightarrow$ & ০০ & 16 \\
\hline TMMC & $0.48(4)$ & $\bullet \bullet \rightarrow$ & $\bullet \circ$ & 17 \\
\hline
\end{tabular}

with $d<d^{*}$, as might occur for example in nanodevices, fluctuation effects become dominant.

The importance of fluctuations in low-dimensional reaction-diffusion processes has also been confirmed experimentally. An effectively one-dimensional setting can be achieved by studying the kinetics of excitons (localized electronic excitations) along polymer chains (other examples are reviewed in [10, 6]). For details, consult the reviews by Kroon and Sprik and Kopelman and Lin in [4. The only purpose of the polymer chains is to provide a carrier for the excitons. Schematically, single excitons may hop from one monomer to the next (thus modelling a diffusive motion) while a reaction occurs if two excitons meet. One may have one or both of the reactions $\bullet \bullet \rightarrow \infty$ or $\bullet \bullet \bullet \circ$, see table 1 We shall show in section 6 that for any branching ratio $B=\Gamma(\bullet \bullet \rightarrow \circ) / \Gamma(\bullet \bullet \rightarrow \bullet)$ the long-time behaviour is still described by the model (1), with a renormalized rate. For late times, one expects the mean exciton density to fall off as a power law $\bar{n}(t) \sim t^{-y}$. The excitons are unstable, with lifetimes of the order $\lesssim 10^{-3} \mathrm{~s}$. Their decay produces light of a characteristic frequency whose intensity can be used to measure $\bar{n}(t)$ while light with a different frequency is emitted if excitons decay through a pair reaction. This allows to measure $\partial_{t} \bar{n}(t)$ as well through time-resolved experiments down to the picosecond scale. Table 1 gives some results for the exponent $y$ in different materials (the branching ratio $B \lesssim 10 \%$ in the first two lines of table (1). Clearly $y \simeq 1 / 2$ as expected from (6) and far from unity. This is strong evidence in favour of strong fluctuation effects in these systems and against their description through a reaction-diffusion equation (2).

Another aspect becomes apparent if we now briefly consider the triple 
annihilation process $\bullet \bullet \bullet \circ \circ \circ$ combined with single particle diffusion. The reaction-diffusion equation reads

$$
\partial_{t} n(\boldsymbol{r}, t)=D \boldsymbol{\nabla}^{2} n(\boldsymbol{r}, t)-\lambda n(\boldsymbol{r}, t)^{3}
$$

Following the same lines as above, but using now the Hölder inequality, the differential inequality $\partial_{t} \bar{n}(t) \leq-\lambda \bar{n}(t)^{3}$ leads to the bound

$$
\bar{n}(t) \leq \frac{n_{0}}{\sqrt{1+2 n_{0}^{2} \lambda t}} \leq \frac{1}{\sqrt{2 \lambda}} t^{-1 / 2}
$$

This of the same order of magnitude as the long-time behaviour expected from diffusive motion in one spatial dimension. Therefore, and in agreement with scaling arguments showing that $d^{*}=1$ [18, already for the triple annihilation process, fluctuation effects should not play a major role in any physically realizable dimension $(d>1) .^{2}$

In conclusion, the description of reaction-diffusion processes with pair reactions in low dimensions requires a truly microscopic approach beyond kinetic reaction-diffusion equations while these equations may well turn out to be adequate for multiparticle reactions. For that reason, we shall in the following consider the master equation formulation of reaction-diffusion processes with pair-reaction terms only.

\section{Quantum Hamiltonian formulation}

We now review the reformulation of a non-equilibrium stochastic system defined by some master equation in terms of the spectral properties of an associated quantum Hamiltonian $H$ and which goes back at least to the classic paper by Glauber [20]. To be specific, we consider in this section only systems defined on a chain with $L$ sites and two allowed states per site. We represent the states of the system in terms of spin configurations $\boldsymbol{\sigma}=\left\{\sigma_{1}, \sigma_{2}, \ldots, \sigma_{L}\right\}$ where $\sigma=+1$ corresponds to an empty site and $\sigma=-1$ corresponds to a site occupied by a single particle. We are interested in

\footnotetext{
${ }^{2}$ This does not imply however, that models containing both binary and multisite reaction terms could not have a non-trivial behaviour. For example, the phase structure of the pair contact process $(\bullet \bullet \rightarrow \circ, \bullet \bullet \circ \rightarrow \bullet \bullet \bullet)$ with single particle diffusion $(\bullet \circ \leftrightarrow \circ \bullet)$ is presently controversial and under very active study [19].
} 
the probability distribution function $P(\boldsymbol{\sigma} ; t)$ of the configurations $\boldsymbol{\sigma}$. Our starting point is the master equation for the $P(\boldsymbol{\sigma} ; t)$

$$
\partial_{t} P(\boldsymbol{\sigma} ; t)=\sum_{\boldsymbol{\tau} \neq \boldsymbol{\sigma}}[w(\boldsymbol{\tau} \rightarrow \boldsymbol{\sigma}) P(\boldsymbol{\tau} ; t)-w(\boldsymbol{\sigma} \rightarrow \boldsymbol{\tau}) P(\boldsymbol{\sigma} ; t)]
$$

where $w(\boldsymbol{\tau} \rightarrow \boldsymbol{\sigma})$ are the transition rates between the configurations $\boldsymbol{\tau}$ and $\boldsymbol{\sigma}$ and are assumed to be given from the phenomenology. In order to rewrite this as a matrix problem, one introduces a state vector

$$
|P\rangle=\sum_{\boldsymbol{\sigma}} P(\boldsymbol{\sigma} ; t)|\boldsymbol{\sigma}\rangle
$$

and eq. (10) becomes

$$
\partial_{t}|P\rangle=-H|P\rangle
$$

where the matrix elements of $H$ are given by

$$
\langle\sigma|H| \tau\rangle=-w(\boldsymbol{\tau} \rightarrow \boldsymbol{\sigma})+\delta_{\sigma, \tau} \sum_{\boldsymbol{v}} w(\boldsymbol{\tau} \rightarrow \boldsymbol{v})
$$

The operator $H$ describes a stochastic process since all the elements of the columns add up to zero. Conservation of probability $\sum_{\boldsymbol{\sigma}} P(\boldsymbol{\sigma} ; t)=1$ is equivalent to the relation

$$
\langle s| H=0
$$

where $\langle s|=\sum_{\boldsymbol{\sigma}}\langle\boldsymbol{\sigma}|$ is a left eigenvector of $H$ with eigenvalue 0 . Correspondingly, $H$ has at least one right eigenvector $|s\rangle=\sum_{\boldsymbol{\sigma}} P_{s}(\boldsymbol{\sigma})|\boldsymbol{\sigma}\rangle$ with eigenvalue 0 , that is $H|s\rangle=0$. Such a vector does not evolve in time and therefore corresponds to a steady-state distribution of the system. Since in general $H$ is not symmetric, this steady-state vector may be highly non-trivial. Note that all this is completely general and applies to any stochastic process defined by a master equation. With a view on the processes to be studied below one calls $H$ a quantum Hamiltonian and this formulation of the master equation the quantum Hamiltonian formalism (see [6, 10] for recent reviews). The reason for this choice of language is the fact that for the processes studied below (and, in fact, many other processes as well) $H$ is the Hamiltonian of some quantum system such as the Heisenberg XXZ Hamiltonian. The steady-state $|s\rangle$ of a stochastic system corresponds in this mapping to the ground state of the quantum system. The probabilistic interpretation is guaranteed by the 
following classical result.

Theorem 1 (Hyver, Keizer, Schnackenberg [21]): For a quantum Hamiltonian $H$ which satisfies the master equation (12) and has $\langle s|=\sum_{\boldsymbol{\sigma}}\langle\boldsymbol{\sigma}|$ as a left eigenstate such that $\langle s| H=0$, the following holds. (i) There is a stationary state

$$
|s\rangle=\sum_{\boldsymbol{\sigma}} P_{s}(\boldsymbol{\sigma})|\boldsymbol{\sigma}\rangle
$$

such that $H|s\rangle=0$. (ii) Consider the eigenvalue problem $H|n\rangle=E_{n}|n\rangle$, with $n=0,1,2 \ldots$ Then

$$
\text { Re } E_{n} \geq E_{0}=0
$$

(iii) Let $\left|P_{0}\right\rangle=|P(0)\rangle$ be the initial state such that the weights of the individual configurations satisfy $0 \leq P(\boldsymbol{\sigma} ; 0) \leq 1$ and $\left\langle s \mid P_{0}\right\rangle=1$. Then for all times $t \geq 0$, one has

$$
0 \leq P(\boldsymbol{\sigma} ; t) \leq 1 \quad \text { and } \quad\langle s \mid P\rangle=1
$$

(iv) Let $H: \mathbb{R}^{n} \rightarrow \mathbb{R}^{n}$ be a linear map such that for the elements $H_{i j}$ of $H$ holds

$$
H_{i j} \leq 0, \sum_{i=1}^{n} H_{i j}=0 \quad \forall j \in\{1, \ldots, n\}
$$

Then $H$ is a "quantum Hamiltonian" of a Markov process described by the master equation (12).

Time-dependent averages of an observable $F$ are given by the matrix element

$$
\left\langle F>(t)=\sum_{\boldsymbol{\sigma}} F(\boldsymbol{\sigma}) P(\boldsymbol{\sigma} ; t)=\langle s|F| P\rangle=\left\langle s|F \exp (-H t)| P_{0}\right\rangle\right.
$$

and we see that eq. (16) means that the system indeed evolves towards the steady-state $|s\rangle$, thus $P_{s}(\boldsymbol{\sigma})=P(\boldsymbol{\sigma} ; \infty)$.

In what follows, we shall be mainly interested in averages of particle numbers $n_{j}$ at site $j$ and their correlators. These can be expressed in the quantum spin formulation in terms of the projector

$$
\tilde{n}_{j}=\frac{1}{2}\left(1-\sigma_{j}^{z}\right)=\left(\begin{array}{ll}
0 & 0 \\
0 & 1
\end{array}\right)_{j}
$$


and one-point and two-point functions of the $n_{j}$ are then expressed $\mathrm{as}^{3}$

$$
C_{1}(j ; t)=\left\langle\tilde{n}_{j}>(t)=\left\langle s\left|\tilde{n}_{j}\right| P\right\rangle \quad, \quad C_{2}(j, \ell ; t)=\left\langle\tilde{n}_{j} \tilde{n}_{\ell}>(t)=\left\langle s\left|\tilde{n}_{j} \tilde{n}_{\ell}\right| P\right\rangle\right.\right.
$$

Two basic situations are readily distinguished from the spectrum of $H$. If in the limit of infinite lattice size $L \rightarrow \infty$ the lowest excited states have a finite gap $\Gamma$ to the ground state energy $E_{0}=0$, then the averages (21) will approach their steady state values exponentially fast on the time-scale $\tau=1 / \Gamma$. On the other hand, if there is in the $L \rightarrow \infty$ limit a continuous spectrum down to $E_{0}=0$, one expects an algebraic decay of the correlators as the system approaches the steady state.

\section{Hecke algebra and integrability}

Before we shall write down quantum Hamiltonians for certain reaction-diffusion systems explicitly, we need some background information on Hecke algebras in order to make contact with integrability. The Hecke algebra $H_{n}(q)$ is spanned by $n$ generators $e_{i}$

$$
H_{n}(q)=\left\{e_{1}, e_{2}, \ldots, e_{n}\right\}
$$

which satisfy the following relations

$$
\begin{aligned}
e_{i} e_{i \pm 1} e_{i}-e_{i} & =e_{i \pm 1} e_{i} e_{i \pm 1}-e_{i \pm 1} \\
e_{i} e_{j} & =e_{j} e_{i} ; \quad \text { if }|i-j| \geq 2 \\
e_{i}^{2} & =\left(q+q^{-1}\right) e_{i}
\end{aligned}
$$

where $q \in \mathbb{C}$ is a parameter. The representations of $H_{n}(q)$ and the relationship to equilibrium statistical mechanics are discussed in great detail in 22 . We are not only interested in $H_{n}(q)$, but also in some quotients, denoted by $(P, M) H_{n}(q)$ [23]. Two specific examples will be of interest to us. The first such quotient is the celebrated Temperley-Lieb algebra $(2,0) H_{n}(q)$, where in addition to eq. (23) the additional relations

$$
e_{i} e_{i \pm 1} e_{i}-e_{i}=0, e_{i \pm 1} e_{i} e_{i \pm 1}-e_{i \pm 1}=0
$$

\footnotetext{
${ }^{3}$ We stress that the structure of these matrix elements is quite distinct from expectation values $\langle 0|F| 0\rangle$ in ordinary quantum mechanics.
} 
hold. On the other hand, the quotient $(1,1) H_{n}(q)$ is defined through the condition 23 .

$$
\left(e_{i} e_{i+2}\right) e_{i+1}\left(q+q^{-1}-e_{i}\right)\left(q+q^{-1}-e_{i+2}\right)=0 ; i=1,2, \ldots
$$

For the definition of more general quotients, we refer to [23].

Consider the $N \times N$ matrices $E^{a b}$ where $a, b=0,1, \ldots, N-1$. The only non-vanishing element of $E^{a b}$ is the one on the $a^{\text {th }}$ line and the $b^{\text {th }}$ column and this element is equal to unity. Define further

$$
E_{i}^{a b}=\mathbf{1} \otimes \cdots \otimes \mathbf{1} \otimes E^{a b} \otimes \mathbf{1} \otimes \cdots \otimes \mathbf{1}
$$

where $E^{a b}$ occurs on position $i$ and $i=1, \ldots, L$ runs over the sites of a chain. Explicit realizations of the Hecke algebra or one of its quotients may be found in the Perk-Shultz models 24], whose Hamiltonian is of the form

$$
H^{(P, M)}=\sum_{i=1}^{L-1} e_{i}^{(P, M)}
$$

The importance of this observation becomes clear from the following

Theorem 2 (Jones [25]): If $H=\sum_{i=1}^{L-1} e_{i}$, where the $e_{i}$ are the generators of the Hecke algebra $H_{L-1}(q), H$ is integrable through the Baxterization procedure.

The Baxterization procedure allows to define, starting from $H$, in a systematic way Boltzmann weights which satisfy the Yang-Baxter equation. We shall illustrate this in the example of the seven-vertex model in section 5 .

In many practical applications, the following result is useful.

Theorem 3 (Martin, Rittenberg [23]): If $H=\sum_{i=1}^{L-1} e_{i}$, where the $e_{i}$ are the generators of the Hecke algebra quotient $(P, M) H_{L-1}(q)$ and furthermore $H^{\prime}=\sum_{i=1}^{L-1} f_{i}$, where the $f_{i}$ are different generators of the same quotient $(P, M) H_{L-1}(q)$, then $H$ and $H^{\prime}$ have the same eigenvalues, up to degeneracies.

We finish this section by writing explicit examples for the quotients $(2,0)$ and $(1,1)$ in the case $N=2$ [26]. Then the matrices $E^{a b}$ can be expressed 
through Pauli matrices

$$
\sigma^{x}=\left(\begin{array}{ll}
0 & 1 \\
1 & 0
\end{array}\right) \quad, \quad \sigma^{y}=\left(\begin{array}{cc}
0 & -\mathrm{i} \\
\mathrm{i} & 0
\end{array}\right) \quad, \quad \sigma^{z}=\left(\begin{array}{cc}
1 & 0 \\
0 & -1
\end{array}\right)
$$

and we define $\sigma_{i}^{x, y, z}$ by analogy with (26). Set

$$
e_{i}^{(2,0)}=-\frac{1}{2}\left[\sigma_{i}^{x} \sigma_{i+1}^{x}+\sigma_{i}^{y} \sigma_{i+1}^{y}+\frac{q+q^{-1}}{2}\left(\sigma_{i}^{z} \sigma_{i+1}^{z}-1\right)-\frac{q-q^{-1}}{2}\left(\sigma_{i}^{z}-\sigma_{i+1}^{z}\right)\right]
$$

Therefore, the Hamiltonian [27, 28]

$$
H^{(2,0)}=\sum_{i=1}^{L-1} e_{i}^{(2,0)}
$$

is integrable. In addition, it satisfies a quantum group invariance, since

$$
\left[H^{(2,0)}, S^{z}\right]=\left[H^{(2,0)}, S^{ \pm}\right]=0
$$

where, recalling also that $\sigma^{ \pm}=\frac{1}{2}\left(\sigma^{x} \pm \mathrm{i} \sigma^{y}\right)$

$$
\begin{aligned}
S^{z} & =\frac{1}{2} \sum_{i=1}^{L} \sigma_{i}^{z}, S^{ \pm}=\sum_{i=1}^{L} S_{i}^{ \pm} \\
S_{i}^{ \pm} & =\exp \left(\frac{\ln q}{2} \sum_{\ell=0}^{i-1} \sigma_{\ell}^{z}\right) \sigma_{i}^{ \pm} \exp \left(-\frac{\ln q}{2} \sum_{\ell=i+1}^{L} \sigma_{\ell}^{z}\right)
\end{aligned}
$$

which in turn obey the commutation relations of $U_{q}(s u(2))$, namely

$$
\left[S^{z}, S^{ \pm}\right]= \pm S^{ \pm} \quad, \quad\left[S^{+}, S^{-}\right]=\frac{q^{2 S^{z}}-q^{-2 S^{z}}}{q-q^{-1}}
$$

On the other hand, set

$$
e_{i}^{(1,1)}=-\frac{1}{2}\left[\sigma_{i}^{x} \sigma_{i+1}^{x}+\sigma_{i}^{y} \sigma_{i+1}^{y}+q \sigma_{i}^{z}+q^{-1} \sigma_{i+1}^{z}-q-q^{-1}\right]
$$

Here, the associated integrable Hamiltonian [29] $H^{(1,1)}=\sum_{i=1}^{L-1} e_{i}^{(1,1)}$ in addition is invariant under the supersymmetric quantum group $U_{q}(s u(1 \mid 1))$, since

$$
\left[H^{(1,1)}, S^{z}\right]=\left[H^{(1,1)}, T^{ \pm}\right]=0
$$


where

$$
T^{ \pm}=q^{(1-L) / 2} \sum_{i=1}^{L} q^{i-1} \exp \left(\frac{\mathrm{i} \pi}{2} \sum_{\ell=1}^{i-1}\left(\sigma_{\ell}^{z}+1\right)\right) \sigma_{i}^{ \pm}
$$

and

$$
\left[S^{z}, T^{ \pm}\right]= \pm T^{ \pm},\left\{T^{+}, T^{-}\right\}=\frac{q^{L}-q^{-L}}{q-q^{-1}}
$$

\section{Single-species models}

We are now ready to study explicit examples of stochastic quantum Hamiltonians. The classical example merely considers particles of a single species $(\bullet)$ which may hop randomly onto an empty nearest-neighbour site (o), thereby modelling the reversible reaction $\bullet \circ \leftrightarrow \circ \bullet$ with rate $D$. This process is often called the symmetric exclusion process. The quantum Hamiltonian reads

$$
H=-\frac{D}{2} \sum_{j=1}^{L-1}\left[\sigma_{j}^{x} \sigma_{j+1}^{x}+\sigma_{j}^{y} \sigma_{j+1}^{y}+\left(\sigma_{j}^{z} \sigma_{j+1}^{z}-1\right)\right]
$$

and coincides with the (ferromagnetic) XXX Heisenberg quantum chain [30].

Certainly, one may now use the Bethe ansatz solution of $H_{\mathrm{Xxx}}$ to rederive known results on simple diffusion. The recent interest in this setup comes from the insight that the integrability of the associated quantum chains allow to make contact with the pre-established algebraic techniques for the treatment of these [26, 31]. Independently, integrability was also observed to occur in the transfer matrices for discrete-time dynamics [32, 33]. The enormous possibilities for non-trivial applications then triggered an ongoing wave of activity, see e.g. [2, 4, 10, 26, 34, 35, 36, 37, 38, 39] and references therein.

Following [26, 31, we now give more examples of integrable quantum Hamiltonians of stochastic systems, restricting ourselves for simplicity to a single species of particles and to binary reactions only (see section 1). The reaction rates are defined in table 2, using the convention of various authors, but unfortunately there is no standard notation. While I prefer a light notation (slightly modified from [40]) and shall use it here, ${ }^{4}$ other

\footnotetext{
${ }^{4}$ The letter $\nu$ is inspired by $\mathbf{n}$ aissance (French for birth) and $\sigma$ comes from $\mathbf{S}$ chöpfung (German for creation). The letter $\beta$ might have come from branching.
} 


\begin{tabular}{|l|l|l|l|l|l|l|}
\hline diffusion to the left & $\circ \bullet \rightarrow \bullet$ & $D_{L}$ & $a_{32}$ & $w_{32}$ & $w_{1,1}(1,0)$ & $\Gamma_{10}^{01}$ \\
diffusion to the right & $\bullet \bullet \rightarrow \circ \bullet$ & $D_{R}$ & $a_{23}$ & $w_{23}$ & $w_{1,1}(0,1)$ & $\Gamma_{01}^{10}$ \\
pair annihilation & $\bullet \bullet \rightarrow \circ \circ$ & $2 \alpha$ & $a_{14}$ & $w_{14}$ & $w_{1,1}(0,0)$ & $\Gamma_{00}^{11}$ \\
coagulation to the right & $\bullet \bullet \rightarrow \circ \bullet$ & $\gamma_{R}$ & $a_{24}$ & $w_{24}$ & $w_{1,0}(0,1)$ & $\Gamma_{01}^{11}$ \\
coagulation to the left & $\bullet \bullet \rightarrow \bullet \circ$ & $\gamma_{L}$ & $a_{34}$ & $w_{34}$ & $w_{0,1}(1,0)$ & $\Gamma_{10}^{11}$ \\
death at the left & $\bullet \bullet \rightarrow \circ \circ$ & $\delta_{L}$ & $a_{13}$ & $w_{13}$ & $w_{1,0}(0,0)$ & $\Gamma_{00}^{10}$ \\
death at the right & $\circ \bullet \rightarrow \circ \circ$ & $\delta_{R}$ & $a_{12}$ & $w_{12}$ & $w_{0,1}(0,0)$ & $\Gamma_{00}^{01}$ \\
decoagulation to the left & $\circ \bullet \rightarrow \bullet \bullet$ & $\beta_{L}$ & $a_{42}$ & $w_{42}$ & $w_{1,0}(1,1)$ & $\Gamma_{11}^{01}$ \\
decoagulation to the right & $\bullet \rightarrow \bullet \bullet$ & $\beta_{R}$ & $a_{43}$ & $w_{43}$ & $w_{0,1}(1,1)$ & $\Gamma_{11}^{10}$ \\
birth at the right & $\circ \rightarrow \circ \bullet$ & $\nu_{R}$ & $a_{21}$ & $w_{21}$ & $w_{0,1}(0,1)$ & $\Gamma_{01}^{00}$ \\
birth at the left & $\circ \rightarrow \bullet \circ$ & $\nu_{L}$ & $a_{31}$ & $w_{31}$ & $w_{1,0}(1,0)$ & $\Gamma_{10}^{00}$ \\
pair creation & $\circ \rightarrow \bullet \bullet$ & $2 \sigma$ & $a_{41}$ & $w_{41}$ & $w_{1,1}(1,1)$ & $\Gamma_{11}^{00}$ \\
\hline Rates defined after reference & {$[40]$} & {$[35]$} & {$[10]$} & {$[31]$} & {$[41]$} \\
\hline
\end{tabular}

Table 2: Two-sites reaction-diffusion processes of a single species and their rates as denoted by various authors.

authors often opt for a systematic, though heavier notation with several indices.

For the time being and for purposes of illustration let us consider besides diffusion only those reactions which irreversibly reduce the number of particles (that is, $\beta_{L, R}=\nu_{L, R}=\sigma=0$ ). Define

$$
\begin{gathered}
D=\sqrt{D_{L} D_{R}}, \gamma=\frac{\sqrt{\gamma_{L} \gamma_{R}}}{D}, \delta=\frac{\sqrt{\delta_{L} \delta_{R}}}{D}, q=\sqrt{\frac{D_{L}}{D_{R}}}=\sqrt{\frac{\gamma_{L}}{\gamma_{R}}}=\sqrt{\frac{\delta_{L}}{\delta_{R}}} \\
\Delta=\frac{1}{2}\left(q+q^{-1}\right)(1+\delta-\gamma)-\alpha / D, h=\frac{1}{2}\left(2 \alpha / D+\gamma\left(q+q^{-1}\right)\right) .
\end{gathered}
$$

Note that the ratio of the left and right rates is taken to be the same for diffusion, coagulation and death processes. We first consider an open chain with $L$ sites. Then the quantum Hamiltonian becomes

$$
H=D\left(H_{\mathrm{XXZ}}(h, \Delta, \delta)+H_{\alpha}+H_{\gamma}+H_{\delta}\right)
$$

where $H_{\mathrm{XxZ}}(h, \Delta, \delta)$ is the standard XXZ quantum chain, including bulk and boundary magnetic fields

$$
H_{\mathrm{XXZ}}(h, \Delta, \delta)=-\frac{1}{2} \sum_{j=1}^{L-1}\left[\sigma_{j}^{x} \sigma_{j+1}^{x}+\sigma_{j}^{y} \sigma_{j+1}^{y}+\Delta\left(\sigma_{j}^{z} \sigma_{j+1}^{z}-1\right)\right.
$$




$$
\left.+h\left(\sigma_{j}^{z}+\sigma_{j+1}^{z}-2\right)-\frac{1}{2}(1-\delta)\left(q-q^{-1}\right)\left(\sigma_{j}^{z}-\sigma_{j+1}^{z}\right)\right]
$$

which contains the diagonal and diffusive matrix elements while the particle annihilation terms are contained in

$$
\begin{aligned}
& H_{\alpha}=-2 \alpha \sum_{j=1}^{L-1} q^{-2 j-1} \sigma_{j}^{+} \sigma_{j+1}^{+} \\
& H_{\gamma}=-\gamma \sum_{j=1}^{L-1} q^{-j}\left(\tilde{n}_{j} \sigma_{j+1}^{+}+q^{-1} \sigma_{j}^{+} \tilde{n}_{j+1}\right) \\
& H_{\delta}=-\delta \sum_{j=1}^{L-1} q^{-j}\left(q^{-2}\left(1-\tilde{n}_{j}\right) \sigma_{j+1}^{+}+q \sigma_{j}^{+}\left(1-\tilde{n}_{j+1}\right)\right)
\end{aligned}
$$

and $\sigma^{ \pm}=\frac{1}{2}\left(\sigma^{x} \pm \mathrm{i} \sigma^{y}\right)$ are the one-particle annihilation/creation operators.

For a physical understanding of this we consider two special cases.

1. Consider pure asymmetric diffusion, that is $\alpha=\gamma=\delta=0$, also referred to as the asymmetric exclusion process. Then $H=D H^{(2,0)}$ as given by eqs. (2930). We thus have a very clear physical interpretation of the quantum-group parameter $q=\sqrt{D_{R} / D_{L}}$ [31]. Besides simple biased diffusion, this model is related e.g. to the $1 D$ Kardar-ParisiZhang equation or to the noisy Burgers equation [42]. The quantum group may be used for the calculation of correlation functions [43].

2. In addition to diffusion, add annihilation such that $2 \alpha=D_{L}+D_{R}$, that is $\Delta=0$ and keep $\gamma=\delta=0$. Then $H=D\left(H_{0}+H_{1}\right)$, where the hermitian part $H_{0}=H^{(1,1)}$ is given by eq. (34) and this part alone is therefore supersymmetric. On the other hand, $H=D \sum_{i=1}^{L-1} f_{i}$, where $f_{i} \in(1,1) H_{L-1}(q)$, but the $f_{i}$ are no longer symmetric. This was the first example of a non-symmetric realization of a Hecke algebra [31]. We remark that besides the already established integrability, this system is also soluble through free-fermion techniques.

Proposition 1 [31]: The spectrum of $H$ in eq. [41) is independent of the particle-reaction terms contained in eq. 43), that is

$$
\operatorname{spec}(H)=\operatorname{spec}\left(D H_{\mathrm{XxZ}}(h, \Delta, \delta)\right)
$$


To see this, recall that the XXZ Hamiltonian conserves the number of particles while the reaction terms irreversibly decrease the total particle number. Thus, $H$ can be written in a block diagonal form

$$
H=\left(\begin{array}{ccccc}
\mathcal{N}_{0} & X_{\delta} & X_{\alpha} & & \\
& \mathcal{N}_{1} & X_{\gamma, \delta} & X_{\alpha} & \\
& & \mathcal{N}_{2} & X_{\gamma, \delta} & \ddots \\
& & & \ddots & \ddots
\end{array}\right)
$$

where $\mathcal{N}_{n}$ refers to the $n$-particle states and $X$ are the reaction matrix elements. Because of the identity

$$
\operatorname{det}\left(\begin{array}{cc}
\mathcal{A} & X \\
0 & \mathcal{B}
\end{array}\right)=\operatorname{det} \mathcal{A} \operatorname{det} \mathcal{B}
$$

it follows that the elements of (43) do not enter into the characteristic polynomial of $H$.

Therefore, the phase diagram for the full Hamiltonian $H$ can be read off from the well-known spectrum of $H_{\mathrm{XXz}}(h, \Delta, \delta)$ [44. For our purposes, we need the following [31]. From (40), only the portion of the phase diagram where $h+\Delta \geq 1$ is important for us. First, he spectrum always has a finite gap when $h+\Delta>1$, which is realized whenever $\delta \neq 0$ or $q \neq 1$. Then the ground state of $H_{\mathrm{XXz}}$ is a trivial ferromagnetic frozen state which corresponds to the empty state $\circ \circ \ldots \circ \circ$. The energy gap $\Gamma=E_{1}-E_{0}$ is finite. Second, the spectrum is gapless for $\Delta+h=1$, where the system undergoes a Pokrovsky-Talapov transition. This situation occurs for $\delta=0$ and $q=1$. We have thus identified the cases where the model approaches the steady state exponentially (non-vanishing gap) or algebraically (gapless).

At this point, it is of interest to discuss the rôle of the boundary conditions and we consider now a periodic chain, for simplicity just for the asymmetric exclusion process (that is $\alpha=\gamma=\delta=0$ ). We stress that if $q \neq 1, H_{\text {per }}$ cannot be read from (41) by simply taking periodic boundary conditions. Rather, we have

$$
H_{\mathrm{per}}=-\frac{1}{q+q^{-1}} \sum_{i=1}^{L}\left[q \sigma_{i}^{+} \sigma_{i+1}^{-}+q^{-1} \sigma_{i}^{-} \sigma_{i+1}^{+}+\frac{q+q^{-1}}{4}\left(\sigma_{i}^{z} \sigma_{i+1}^{z}-1\right)\right]
$$

together with the periodic boundary conditions $\sigma_{L+1}^{ \pm}=\sigma_{1}^{ \pm}$and $\sigma_{L+1}^{z}=\sigma_{1}^{z}$. The hopping terms can be brought back to the familiar XXZ form of eq. (41) 
through a similarity transformation $H_{\text {per }}^{\prime}=\mathcal{U} H_{\text {per }} \mathcal{U}^{-1}$ with the matrix $\mathcal{U}=$ $\exp \left(\pi g \sum_{\ell=1}^{L} \ell \sigma_{\ell}^{z}\right)$ with $q=e^{2 \pi g}$ such that [45]

$$
H_{\mathrm{per}}^{\prime}=-\frac{1}{2\left(q+q^{-1}\right)} \sum_{i=1}^{L}\left[\sigma_{i}^{x} \sigma_{i+1}^{x}+\sigma_{i}^{y} \sigma_{i+1}^{y}+\frac{q+q^{-1}}{2}\left(\sigma_{i}^{z} \sigma_{i+1}^{z}-1\right)\right]
$$

which looks the same as $D H^{(2,0)}$, but we now have the non-periodic boundary conditions $\sigma_{L+1}^{ \pm}=q^{\mp L} \sigma_{1}^{ \pm}, \sigma_{L+1}^{z}=\sigma_{1}^{z}$. As a consequence, $\operatorname{spec}\left(H_{\text {per }}\right)$ has no gap even for $q \neq 1$. While for a finite number $r$ of particles and long chains $L \rightarrow \infty$, it is easy to see that $\operatorname{Re} E_{\text {per }} \sim L^{-2}$ [45], for finite densities $n=r / L$, an elaborate Bethe ansatz calculation shows that $\operatorname{Re} E_{\mathrm{per}} \sim L^{-3 / 2}$ [42. Therefore, and quite in distinction with equilibrium systems, a change in the boundary conditions may well induce a phase transition in the long-time behaviour (observed first in driven diffusive systems [46]). What happens is easily understood in this particular example. For an open chain, the particles get stuck at one end of the chain and a non-trivial position-dependent steadystate density-profile $\rho_{s}(i)$ builds up. The time needed for this should be of the order of the time the particles need to move from one end to the other which is finite for $q \neq 1$. On the other hand, for a periodic chain the particles keep chasing other forever and a steady-state particle current will be observed. By going to a reference frame co-moving with the mean velocity of that current, one is back to the case $q=1$ of unbiased diffusion.

The energy gaps $\Gamma$ can now be found by concentrating on the spectrumgenerating part $H_{\mathrm{Xxz}}$. For $h+\Delta>1$, the gaps are finite and are easily found. We concentrate here on the case of unbiased diffusion, when $h+\Delta=1$ and we are at the Pokrovsky-Talapov transition. The low-lying energy gaps are given by the following

Proposition 2: On the Pokrovsky-Talapov line $h+\Delta=1, \delta=0$, and for $L$ large, the low-lying eigenvalues of $H$ eq. 41) are for periodic boundary conditions

$$
E_{r}^{(\mathrm{per})}=D\left(\frac{2 \pi}{L}\right)^{2}\left(I_{1}^{2}+\ldots+I_{r}^{2}\right)-\frac{8 \pi^{3} D}{L^{3}} \frac{\Delta}{1-\Delta} \sum_{j, \ell=1}^{r}\left(I_{j}-I_{\ell}\right)^{2}+O\left(L^{-4}\right)
$$

where the $I_{j}$ are pairwise distinct integers (half-integers) when $r$ is odd (even). For an open chain

$$
E_{r}^{(\text {free })}=D\left(\frac{\pi}{L}\right)^{2}\left(I_{1}^{2}+\ldots+I_{r}^{2}\right) \cdot\left(1-\frac{2(r-1)}{L} \frac{\Delta}{1-\Delta}\right)+O\left(L^{-4}\right)
$$


where the $I_{j}$ are pairwise distinct non-negative integers. The integer $r=$ $0,1,2, \ldots$ gives the number of particles in the sectors of $H_{\mathrm{Xxz}}$.

The finite-size amplitudes $\lim _{L \rightarrow \infty} L^{2} E_{r}$ are independent of $\Delta$, for either periodic or open chains. This proves an old conjecture [31] based on numerical calculations. For $\Delta=0$, the well-known free-fermion solution is reproduced. To leading order in $L^{-1}$, eigenvalues with the same $I_{1}^{2}+\ldots+I_{r}^{2}$ are degenerate. We observe that for periodic boundary conditions, this degeneracy is already broken by the first correction in $1 / L$, while for free boundary conditions, the leading correction keeps that symmetry.

Eqs. (4950) are easily found from the Bethe ansatz. We first consider periodic boundary conditions. The XXZ chain may broken into sectors containing only the states with $r$ particles. Performing the Bethe ansatz as usual [47, one has for the energies

$$
E_{r}=2 D\left(r-\cos k_{1}-\ldots-\cos k_{r}\right)
$$

where the quasimomenta $k_{1}, \ldots, k_{r}$ are solutions of the Bethe ansatz equations

$$
L k_{j}=2 \pi I_{j}-\sum_{\ell=1}^{r} \Theta\left(k_{j}, k_{\ell}\right) ; j=1, \ldots, r
$$

where the $I_{j}$ are pairwise distinct integers (half-integers) when $r$ is odd (even) and

$$
\Theta\left(k, k^{\prime}\right)=2 \arctan \frac{\Delta \sin \left(\left(k-k^{\prime}\right) / 2\right)}{\cos \left(\left(k+k^{\prime}\right) / 2\right)-\Delta \cos \left(\left(k-k^{\prime}\right) / 2\right)}
$$

We are interested in the leading finite-size corrections when $L \rightarrow \infty$ with $r$ fixed. The ansatz

$$
k_{j}=\frac{2 \pi}{L} I_{j}+\frac{a_{j}}{L^{2}}+\ldots
$$

gives $\Theta\left(k_{1}, k_{2}\right) \simeq \frac{\Delta}{1-\Delta} \frac{2 \pi}{L}\left(I_{1}-I_{2}\right)+O\left(L^{-2}\right)$ and $a_{j}=\frac{2 \pi \Delta}{1-\Delta} \sum_{\ell=1}^{r}\left(I_{\ell}-I_{j}\right)$. Then (49) follows. Second, for free boundary conditions, the Bethe ansatz [48] reproduces eq. (511) for the energies, while the Bethe ansatz equations for the quasimomenta now take the form

$$
L k_{j}=\pi I_{j}-\frac{1}{2} \sum_{\ell \neq j}\left[\Theta\left(k_{j}, k_{\ell}\right)-\Theta\left(-k_{j}, k_{\ell}\right)\right]
$$

for $j=1, \ldots, r$ and where the $I_{j}$ are pairwise distinct non-negative integers. Eq. (54) leads to $a_{j}=-\frac{\Delta}{1-\Delta}(r-1) \pi I_{j}^{2}$ and we arrive at (501). q.e.d. 


\section{The seven-vertex model}

Having seen for some single-species reaction-diffusion processes how the relationship with the Bethe-ansatz solution XXZ chain could be used to infer certain physical properties, we present in this section an example of the Baxterization procedure [25]. That procedure permits to associate to a stochastic quantum Hamiltonian $H$ related to a Hecke algebra the Boltzmann weights of a corresponding two-dimensional vertex model and thus prove its integrability. In principle, the model is then solved through the Bethe ansatz. For the six- and eight-vertex models the completeness of the Bethe ansatz has recently been proven [49].

Following [31, we consider the pair-annihilation model eq. (41) already defined in section 4 with $\gamma=\delta=0$ and furthermore, we take $2 \alpha=D_{R}+D_{L}$, thus $\Delta=0$. We call $\Omega=2 \alpha / D$. After having performed the canonical transformation $E_{i}^{a b} \rightarrow(-1)^{a-b} E_{i}^{a b}$, only at even sites $i$, the quantum Hamiltonian takes in the basis given by eq. (28) the simple form

$$
H=-D \sum_{i=1}^{L-1} e_{i}
$$

where

$$
e_{i}=\mathbf{1}_{1} \otimes \ldots \otimes \mathbf{1}_{i-1} \otimes\left(\begin{array}{cccc}
0 & 0 & 0 & \Omega \\
0 & q^{-1} & q & 0 \\
0 & q^{-1} & q & 0 \\
0 & 0 & 0 & q+q^{-1}
\end{array}\right) \otimes \mathbf{1}_{i+2} \otimes \ldots
$$

and $\mathbf{1}_{i}$ are $2 \times 2$ unit matrices attached to the site $i$.

While we could certainly solve this particular model through a JordanWigner transformation followed by a canonical transformation, see e.g. 40, we are interested in generic approaches of a more general validity than in those cases reducible to a free-fermion description.

We have already seen in section 4 that the $e_{i}$ satisfy the Hecke algebra (23). We now construct a two-dimensional vertex model corresponding to $H$ having a row-to-row transfer matrix $T(\theta)$ depending on the spectral parameter $\theta$. That transfer matrices will satisfy the Yang-Baxter equations [50] which imply the commutation relations $\left[T(\theta), T\left(\theta^{\prime}\right)\right]=0$ if $\theta \neq \theta^{\prime}$. The

construction is based on the matrix $\check{R}_{i}(\theta), i=1,2, \ldots, L-1$ which depends 
on the spectral parameter $\theta$. The Baxterization procedure for Hecke algebras 25] gives

$$
\check{R}_{i}(\theta)=\frac{\sinh \theta}{\sinh \eta} e_{i}+\frac{\sinh (\eta-\theta)}{\sinh \eta} ; q=e^{\eta}
$$

which for our model (56 57) leads to

$$
\check{R}_{i}(\theta)=\mathbf{1}_{1} \otimes \ldots \otimes \mathbf{1}_{i-1} \otimes \mathcal{R}_{i, i+1} \otimes \mathbf{1}_{i+2} \otimes \ldots
$$

with the non-vanishing elements of the matrix $\mathcal{R}_{i, i+1}$

$$
\mathcal{R}_{i, i+1}:=\frac{1}{\sinh \eta}\left(\begin{array}{cccc}
\sinh (\eta-\theta) & & & \Omega \sinh \theta \\
& e^{-\theta} \sinh \eta & e^{\eta} \sinh \theta & \\
& e^{-\eta} \sinh \theta & e^{\theta} \sinh \eta & \\
& & & \sinh (\eta+\theta)
\end{array}\right)
$$

The relations eq. (23) imply that these matrices satisfy the spectral parameterdependent braid-group relations

$$
\begin{aligned}
\check{R}_{i}(\theta) \check{R}_{i \pm 1}\left(\theta+\theta^{\prime}\right) \check{R}_{i}\left(\theta^{\prime}\right) & =\check{R}_{i \pm 1}\left(\theta^{\prime}\right) \check{R}_{i}\left(\theta+\theta^{\prime}\right) \check{R}_{i \pm 1}(\theta) \\
{\left[\check{R}_{i}(\theta), \check{R}_{j}\left(\theta^{\prime}\right)\right] } & =0 ;|i-j| \geq 2
\end{aligned}
$$

which are equivalent to the Yang-Baxter equations.

In a $2 D$ vertex model with vertex configurations labelled by $(k, \ell, m, n)$, the Boltzmann weights $S_{\ell, m}^{k n}$ are obtained from

$$
\check{R}_{i}(\theta)=S_{\ell, m}^{k, n} \mathbf{1}_{1} \otimes \ldots \otimes \mathbf{1}_{i-1} \otimes E^{m k} \otimes E^{n \ell} \otimes \mathbf{1}_{i+2} \otimes \ldots
$$

This implies that the vertex model associated to eq. (56) is a seven-vertex model. In a vertex model, arrows are attached to the bonds of a square lattice [50]. In the stochastic model, we associate a particle $(\bullet)$ with an arrow pointing up/right and no particle (o) with an arrow pointing down/left. In figure 2 we list together the chemical reactions, the vertex configurations and their Boltzmann weights. The vertices usually labelled 1 to 4 correspond to no reaction and are not shown (see [31]). Vertices 5 and 6 correspond to diffusion to the right and to the left and vertex 7 to pair-annihilation. In the leftmost column of figure 2, the state of the particles before the reaction is given as the lower pair of symbols while the state after the reaction is given by the upper pair of symbols. The middle column gives the corresponding vertex configuration and the right column the Boltzmann weight. The Hamiltonian eq. (56) may be recovered from $H=-\left.\frac{\mathrm{d}}{\mathrm{d} \theta} \ln T(\theta)\right|_{\theta=0}$. 
Figure 2: Diffusion and pair-annihilation of particles in the seven-vertex model and their Boltzmann weights, for the vertices 5 to 7 .

5.

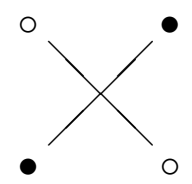

6.

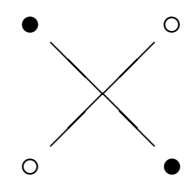

7.

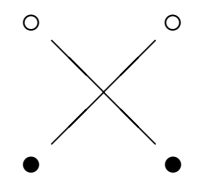

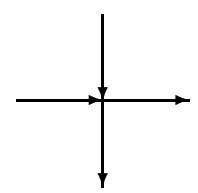
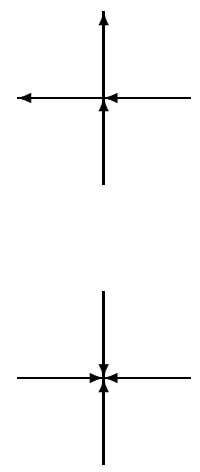

$$
e^{-\eta} \sinh \theta
$$

$e^{\eta} \sinh \theta$

$\Omega \sinh \theta$

\section{$6 \quad$ Further applications}

We have studied in some detail the pair-annihilation process and its integrability. Still, extracting explicitly information about the long-time behaviour (or the steady-state in more complicated models) is not yet trivial. In this section, we briefly review some approaches which may be useful.

A) Spectral integrability. We have already seen that in certain cases, the quantum Hamiltonian $H=H_{0}+H_{1} \operatorname{such}$ that $\operatorname{spec}(H)=\operatorname{spec}\left(H_{0}\right)$, independently of the precise form of $H_{1}$. It may happen that although $H_{0}$ is integrable, $H$ is not. Such a model is said to be spectrally integrable. If only binary interactions are present, it is convenient to express $H$ in terms of a two-site matrix $H_{i, i+1}$ acting on the sites $i$ and $i+1$

$$
H=\sum_{j=1}^{L} \mathbf{1}_{1} \otimes \cdots \otimes \mathbf{1}_{j-1} \otimes H_{j, j+1} \otimes \mathbf{1}_{j+2} \cdots \otimes \mathbf{1}_{L}
$$




\begin{tabular}{|c|cc|}
\hline$\delta$ & $C_{1}(t)$ & $C_{2 ; n}(t)$ \\
\hline 0 & $t^{-1 / 2}$ & $t^{-3 / 2}$ \\
$<\alpha+\gamma$ & $\exp (-2 \delta t)$ & $t^{-1 / 2} \exp (-4 \delta t)$ \\
$>\alpha+\gamma$ & $\exp (-2 \delta t)$ & $\exp [-4 \delta t+2(\Delta+1 / \Delta-2) t]$ \\
\hline
\end{tabular}

Table 3: Generic long-time behaviour of the one-point and two-point correlators $C_{1}(t)$ and $C_{2 ; n}(t)$ for $n$ finite, in the system eq. (64) and with a translation-invariant initial state and a finite initial particle-density.

For the model (41) with left-right symmetry, that is $q=1$, we have

$$
H_{j, j+1}=D\left(\begin{array}{cccc}
0 & -\delta & -\delta & -2 \alpha \\
0 & 1+\delta & -1 & -\gamma \\
0 & -1 & 1+\delta & -\gamma \\
0 & 0 & 0 & 2(\alpha+\gamma)
\end{array}\right)
$$

One can always rescale time such that $D=1$. Then the parameters of the XXZ chain $H_{0}=H_{\mathrm{XXZ}}$ become $\Delta=1+\delta-\gamma-\alpha$ and $h=\alpha+\gamma$.

In eqs. (6364) $H$ is only integrable for $\delta=0$. On the other hand, the special case $\alpha=0, \gamma=\delta$ simply corresponds to the radioactive decay of diffusively moving particles. While for $\delta=0$, one is back to the critical Pokrovsky-Talapov line $h+\Delta=1$, the associated quantum spin chain has a frozen ground state for $\delta=0$. The one-particle and two-particle correlators $C_{1}(t)=\sum_{j=1}^{L} C_{1}(j ; t)$ and $C_{2 ; n}(t)=\sum_{j=1}^{L} C(j, j+n ; t)$ with $n$ fixed, see eq. (21), only imply the sectors with $r=1$ and $r=2$ particles of $H_{\mathrm{XxZ}}$, respectively. Their long-time behaviour is easily worked out from the results of section 4 and is collected in table 3 40. Of course, we implicitly assume that the corresponding amplitudes do not accidentally vanish. At first sight, one might have expected a simple exponential factor $e^{-2 k \delta t}$ for the $k$-point correlator $C_{k}$ and we already observe that eventual algebraic prefactors are not readily predicted from the spectrum of $H$ alone. The more complicated form of the relaxation time for $\delta>\alpha+\gamma$ comes from a bound state in the two-particle sector of $H_{\mathrm{XXZ}}$, with energy $4 \delta+4-2 \Delta-2 / \Delta$, see [50, [51]. More general initial conditions are discussed in [40].

B) Similarity transformations. In trying to extract explicit information on certain reaction-diffusion systems, the integrability of the quantum Hamiltonian $H$ plays a central rôle. Since it is difficult to realize the constraints 
(18) of stochasticity and integrability at the same time, it is of interest to see whether there exist systematic transformations of an integrable quantum Hamiltonian $H$ towards a new stochastic Hamiltonian $\widetilde{H}$.

Specifically, we shall consider the transformation

$$
\widetilde{H}=\mathcal{B H B}^{-1} \quad, \quad \mathcal{B}=\bigotimes_{j=1}^{L} B_{j}
$$

where $B_{j}=\mathbf{1} \otimes \ldots \otimes \mathbf{1} \otimes B \otimes \mathbf{1} \otimes \ldots \otimes \mathbf{1}$ is the transformation matrix $B$ acting on the site $j$. Then the systems described by $H$ and $\widetilde{H}$ are said to be similar to each other. An interesting alternative, which has not yet been systematically studied, is to consider an enantiodromy transformation [10]

$$
\widetilde{H}=\mathcal{B} H^{\mathrm{T}} \mathcal{B}^{-1}
$$

where $H^{\mathrm{T}}$ is the transpose of $H$.

From now on, we shall focus on translationally invariant systems and consider periodic boundary conditions. The effect of the transformation $\mathcal{B}$ on $H$ is completely given by its effect on the two-particle Hamiltonian $H_{j, j+1}$ in (64). A stochastic similarity transformation arises if both $H$ and $\widetilde{H}$ represent stochastic systems. For a simple example, consider the symmetric annihilation-coagulation process eqs. (6364) with $\delta=0$. If $C(t \mid \alpha, \gamma)=C_{1}(t)$ is the spatially averaged particle density with the rates $\alpha$ and $\gamma$, respectively, a stochastic similarity transformation shows that [52, 53, 54]

$$
C_{1}(t \mid \alpha, \gamma)=\frac{\alpha+\gamma}{2 \alpha+\gamma} C_{1}(t \mid 0, \alpha+\gamma)
$$

Similar results hold for any $k$-point correlator $C_{k}(t)$. So far, explicit methods to find the time-dependent correlators are only available for either the pure coagulation model $\alpha=0$ through empty-interval methods (see below) or the pure annihilation model $\gamma=0$ through free-fermion techniques, see [55. 40, 10, and references therein. Eq. (67) allows to reduce any symmetric annihilation-coagulation process to pure coagulation, for any initial density $C_{1}(0 \mid 0, \gamma)$. This also explains the experimental results in table 1 . The known stochastic similarity transformations of the form (65) leave the parameters $\Delta$ and $h$ of the XXZ chain invariant, but the results of Proposition 2 suggest that a stochastic similarity transformation between systems with different values of $\Delta$ might exist. See [53] for the extensions to $\delta>0$ and $q \neq 1$. 


\begin{tabular}{|c|c|c|c|c|}
\hline model & \multicolumn{3}{|c|}{ reactions } & conditions \\
\hline $\mathrm{A}$ & $\bullet \bullet \leftrightarrow \circ ০$ & & $\bullet \leftrightarrow \leftrightarrow \circ \bullet$ & $2(\alpha+\sigma)=D_{L}+D_{R}$ \\
\hline B & $\bullet \bullet \rightarrow \circ ০$ & $\bullet \bullet \rightarrow \diamond \bullet, \bullet \circ$ & $\bullet \circ \leftrightarrow \circ \bullet$ & $2 \alpha+\gamma_{L}+\gamma_{R}=D_{L}+D_{R}$ \\
\hline $\mathrm{C}$ & $\circ \circ \rightarrow \bullet \bullet$ & $\circ \circ \rightarrow \diamond \bullet, \bullet$ & $\bullet \circ \leftrightarrow \circ \bullet$ & $2 \sigma+\nu_{L}+\nu_{R}=D_{L}+D_{R}$ \\
\hline $\mathrm{D}$ & $\bullet \bullet \rightarrow \bullet, \circ \bullet$ & $\bullet \circ, \circ \bullet \rightarrow \bullet \bullet$ & $\bullet \circ \leftrightarrow \circ \bullet$ & $\gamma_{L}=D_{L} \quad \gamma_{R}=D_{R}$ \\
\hline $\mathrm{E}$ & $\circ \circ \rightarrow \bullet \circ, \circ \bullet$ & $\bullet \circ, \circ \bullet \rightarrow \circ \circ$ & $\bullet \circ \leftrightarrow \circ \bullet$ & $\nu_{L}=D_{L} \quad \nu_{R}=D_{R}$ \\
\hline $\mathrm{F}$ & $\bullet \circ, \circ \bullet \rightarrow \circ \circ$ & $\bullet \circ, \diamond \bullet \rightarrow \bullet \bullet$ & & $\delta_{R} / \delta_{L}=\beta_{R} / \beta_{L}$ \\
\hline G & $\bullet \circ, \infty \bullet$ & $\rightarrow \bullet \bullet, \infty \circ$ & & $\begin{array}{ll}\delta_{R}=\beta_{R} & \gamma_{R}=\nu_{L} \\
\delta_{L}=\beta_{L} & \gamma_{L}=\nu_{R}\end{array}$ \\
\hline $\mathrm{H}$ & $\bullet \rightarrow 0$ & $\circ \rightarrow \bullet$ & & $\begin{array}{c}\delta_{L}=\delta_{R}=\gamma_{L}=\gamma_{R} \\
\beta_{L}=\beta_{R}=\nu_{L}=\nu_{R}\end{array}$ \\
\hline
\end{tabular}

Table 4: Single-species processes with space-independent reaction rates and which are similar via (65) to a free-fermion model. The reaction rates are defined in table 2 .

Eq. (67) allows to recover the long-time behaviour $C(t \mid \alpha, \gamma) \sim t^{-1 / 2}$ from a simple heuristic argument. For pure coagulation, one particle always remains, thus $C(\infty \mid 0, \gamma)=1 / L$ in the steady state. Therefore the steady-state density $C(\infty \mid \alpha, \gamma) \sim L^{-1}$. On the other hand, from the spectrum of $H$, the leading relaxation time $\tau=\Gamma^{-1} \sim L^{2} \sim \xi^{2}$, where $\xi$ is identified as the characteristic spatial length scale. Therefore $C(\infty \mid \alpha, \gamma) \sim \xi^{-1} \sim \tau^{-1 / 2}$. The asserted time-dependent behaviour therefore might have been anticipated on dimensional grounds.

C) Free fermions. For the pure annihilation model with $2 \alpha=D_{R}+D_{L}$, one has $\Delta=0$. In this case, $H$ may be diagonalized through a JordanWigner transformation followed by a canonical transformation [55]. In order for this to work, $H$ may only contain pairs of particle creation and annihilation operators. For space-independent reaction rates, the complete list of reaction-diffusion process whose quantum Hamiltonian $\widetilde{H}$ is similar through (65) to a free-fermion Hamiltonian $H$ is as follows [10, 40, 53] and shown in table 4. Since the transformation (65) is spatially local, these correspondences actually hold in any dimension, but free-fermion methods are only available in $1 D$.

Of these models, only models A (diffusive pair-annihilation and creation, solved exactly in $1 D$ in [55]), G (kinetic Ising model with Glauber dynamics) and $\mathrm{H}$ (free decay and creation of particles) are reversible and have an 
equilibrium steady-state. Their quantum Hamiltonian is therefore similar to a symmetric matrix. The similarity of the kinetic Ising model with Glauber dynamics (model G) to a free-fermion model was obtained long ago through a duality transformation [56] and more recently as a true similarity transformation [53, 57]. This suggests to study a more general type of relationship, based on domain-wall dualities, see [10, 57] for details. Models C and E are obtained by a particle-hole permutation $\bullet \leftrightarrow \circ$ from models B and D, respectively. Model B is the biased annihilation-coagulation process, while model $\mathrm{D}$ is the diffusive coagulation process with arbitrary decoagulation. Finally, model F is the doubly biased voter model (in space and in the preference between - and $\circ$ ) and in [58] some correlators are found from the free-fermion form. The physical behaviour of all these models can be treated in a single calculation. For example, the mean particle-density depends on a single parameter $h$ such that 40]

$$
C_{1}(t)-C_{1}(\infty) \sim \begin{cases}t^{-1 / 2} & ; \quad \text { if } h=1 \\ t^{-3 / 2} \exp (-t / \tau) & ; \quad \text { if } 0<h<1\end{cases}
$$

where $C_{1}(\infty)$ is the steady-state density and $\tau=1 /(4-4 h)$ is the relaxation time (see [10] and references therein for more information on solved free-fermion models).

This kind of analysis was generalized to find those reaction-diffusion systems which are similar, via a transformation of the type (65), to the XXZ chain [40]. While the full result is too complex to be re-stated here, an interesting special case is given by the conditions

$$
\begin{aligned}
\gamma_{R}+\beta_{L}+2 \alpha+D_{L} & =\nu_{L}+\delta_{L}+2 \sigma+D_{R} \\
\gamma_{L}+\beta_{R}+2 \alpha+D_{R} & =\nu_{R}+\delta_{R}+2 \sigma+D_{L}
\end{aligned}
$$

In this case the (usually infinite) hierarchy of equations of motion for the $k$ point particle-density correlators $\mathcal{C}_{k}(t)=<n_{1}(t) \ldots n_{k}(t)>$ closes naturally, such that $\dot{\mathcal{C}}_{k}(t)$ only depends on the $\mathcal{C}_{\ell}(t)$ with $\ell \leq k$. In principle the equations of motions for the $\mathcal{C}_{k}$ can then be solved iteratively [35.

D) Partial integrability. The previous sections have shown that constructing integrable stochastic systems which go beyond mere free diffusion is a non-trivial exercice. One might wonder whether the condition of full integrability is not too strong. After all, from a practical point of view it would 
be enough to identify a set $\left\{Q_{1}, \ldots, Q_{M}\right\}$ of observables such that these satisfy a closed set of equations, say $\dot{Q}_{i}=f_{i}\left(Q_{1}, \ldots, Q_{M}\right)$, with $i=1, \ldots, M$. Such a partial integrability may be enough for many practical needs. Indeed, such an approach is available through the empty-interval method [12, 17. Consider a periodic chain with $L$ sites and lattice spacing $a$. Let $I_{n}(t)$ be the probability that at time $t, n$ consecutive sites are empty. Then the mean particle-density is [12, 52 ]

$$
C_{1}(t)=\left(1-I_{1}(t)\right) / a
$$

In order to illustrate the method, we consider the left-right symmetric pure coagulation model and also take the free-fermion condition $\gamma=D$ of model $\mathrm{D}$ in table 4, but we now add a three-site production reaction $\bullet \circ \bullet \rightarrow$ with rate $2 D \lambda\left[59\right.$. The equations of motion for the $I_{n}(t)$ read

$$
\begin{aligned}
& \dot{I}_{1}(t)=2 D\left(I_{0}(t)-2 I_{1}(t)+I_{2}(t)\right)-2 D \lambda\left(I_{1}(t)-2 I_{2}(t)+I_{3}(t)\right) \\
& \dot{I}_{n}(t)=2 D\left(I_{n-1}(t)-2 I_{n}(t)+I_{n+1}(t)\right) ; 2 \leq n \leq L-1
\end{aligned}
$$

together with the boundary conditions $I_{0}(t)=1$ and $I_{L}(t)=0$ (assuming that there is at least one particle in the system). The solution of these equations is straightforward. For example, one obtains for the leading relaxation time $\tau^{-1}=\Gamma=2 D \pi^{2} L^{-2}+O\left(L^{-4}\right)$, in agreement with the results of section 4 . The effect of the production term is only transient, as illustrated in figure 3 for the mean density $C_{1}(t)$. For $\lambda=0, C_{1}(t) \sim 1 / \sqrt{t}$ is of course expected from eqs. (667). While the free-fermion condition $\gamma=D$ is essential for the method to work, we also see that the presence of the production term poses no problem at all for the closure of the equations of motion (171).$^{5}$

Accepting the free-fermion condition $\gamma_{L, R}=D_{L, R}$, one can extend the treatment to the more general model $\mathrm{D}$ of table 4 and may even extend this further to include the processes $\circ \circ \rightarrow \bullet \bullet$ and $\circ \circ \rightarrow \circ \bullet, \bullet \circ$ with rates $2 \sigma, \nu_{R}, \nu_{L}$, respectively [7, 12. Let us call this system model $D$ ' which depends on the seven parameters $D_{L, R}, \beta_{L, R}, \nu_{L, R}, \sigma$. In a remarkable paper [41], the idea of the empty-interval method was translated into the Hamiltonian formalism and several new sets of observables were defined which generalize the variables $I_{n}(t)$ and lead again to closed equations of motion. It turns out that the spectrum of relaxation times of model D' is given by the Hamiltonian

\footnotetext{
${ }^{5}$ This term cannot, e.g. by a similarity transformation, be turned into a term treatable by either free-fermion or full integrability methods.
} 


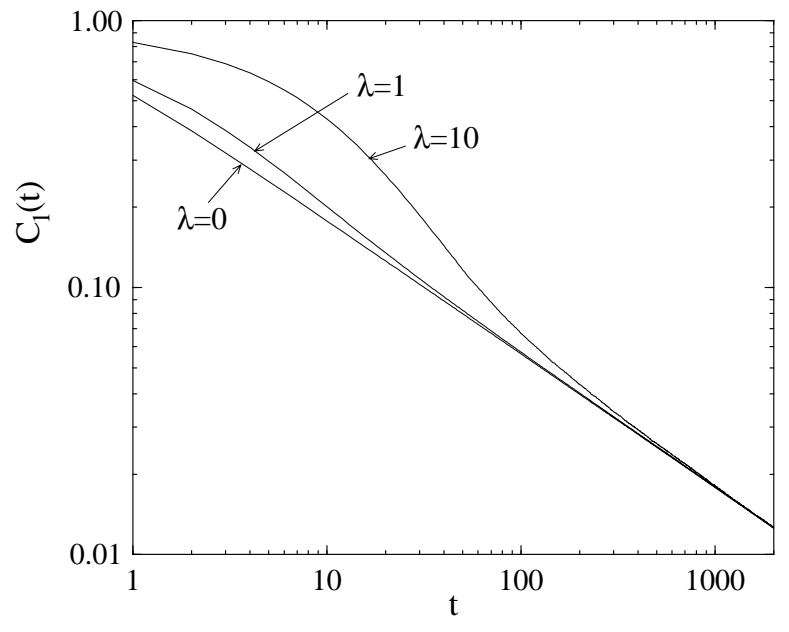

Figure 3: Evolution of the mean particle density $C_{1}(t)$ in the symmetric coagulation model with the production reaction $\bullet \circ \bullet \rightarrow \bullet \bullet \bullet$ for several production rates $\lambda$. For long times, the asymptotic behaviour is $C_{1}(t) \sim 1 / \sqrt{t}$ for all values of $\lambda$ (after [59]).

of the Wannier-Stark ladder [4]

$$
H=-\sum_{n=-L}^{L}\left[\sigma_{n}^{x} \sigma_{n+1}^{x}+\sigma_{n}^{y} \sigma_{n+1}^{y}+\left(h+h^{\prime} n\right) \sigma_{n}^{z}\right]
$$

where $h$ and $h^{\prime}$ are constants. In this case, the couplings in $H$ are spacedependent. The extension of the similarity/enantiodromy approach to this more general setting remains to be done. Extensions of the empty-interval method to interactions on more than two sites are studied in 60.

While the empty-interval method as such does not work for the pairannihilation process, the method has been generalized recently [61]. We briefly explain the idea using the left-right symmetric pair-annihilation process with the free-fermion condition $\alpha=D$ (model A or B) as example. Let $G_{n}(t)$ be the probability that at time $t$, one has on $n$ consecutive sites an even number of particles. The mean particle density is $C_{1}(t)=\left(1-G_{1}(t)\right) / a$. Furthermore, let $F_{n}(t)\left(H_{n}(t)\right)$ be the probability that a segment of $n$ consecutive sites with an even (odd) number of particles is followed by the presence of a particle at the $(n+1)^{\text {th }}$ site. From the relations

$$
2 F_{n}(t)=\left(1-G_{1}\right)+\left(G_{n}-G_{n+1}\right), 2 H_{n}(t)=\left(1-G_{1}\right)-\left(G_{n}-G_{n+1}\right)
$$


and the boundary condition $G_{0}(t)=1$, the equations of motion

$$
\dot{G}_{n}(t)=2 D\left(F_{n-1}-H_{n-1}+H_{n}-F_{n}\right)=2 D\left(G_{n-1}(t)-2 G_{n}(t)+G_{n+1}(t)\right)
$$

follow. They can be solved by standard methods. Reaction terms parametrized by $\sigma, \nu_{L, R}, \beta_{L, R}$ (see table 2) and even the reaction $\circ \bullet \circ \rightarrow \bullet \bullet \bullet$ can be added 61. Correlators are studied in [62].

In view of the practical success of these techniques it is perhaps not completely futile to ask whether there might a be a systematic way to identify these 'empty-interval' or related variables?

E) Multi-species models. We consider chains with $N$ states per site. One of them is taken to be the empty site (o) and the other states are labelled $A_{n}, n=1, \ldots, N-1$. Finding integrable stochastic systems becomes more difficult when $N$ increases. Several examples were found [26] through the quotients $(P, M) H_{L-1}(q)$ as realized through the Perk-Shultz model. They are collected in table 5. The following conventions apply.

1. For the first reaction in all models and the second reaction in models $\mathfrak{B}, \mathfrak{C}, \mathfrak{D}$ (with $n<m$ understood) the reaction to the right (left) occurs with rate $\Gamma_{R}\left(\Gamma_{L}\right)$.

2. For models $\mathfrak{E}, \mathfrak{F}$ the sum $r+s$ has to be taken $\bmod N$. If in this case $r+s=0 \bmod N$, the rate is $\Gamma_{L}+\Gamma_{R}$. If $r+s \neq 0 \bmod N$ as well as for the third reaction in models $\mathfrak{C}, \mathfrak{D}$ the rate is $\Gamma_{R}$. In model $\mathfrak{D}$, it is also assumed that in the third reaction, pairs $(r, s=r \pm 1)$ never have an element in common. If the products on the right are interchanged, (e.g. $A_{1} A_{1} \rightarrow A_{1} \circ$ in model $\mathfrak{C}$ ), the rate is $\Gamma_{L}$.

3. In the third reaction in model $\mathfrak{F}$ the rates are $\Gamma_{ \pm}$, respectively such that $\Gamma_{+}+\Gamma_{-}=\Gamma_{L}+\Gamma_{R}$.

One defines $D=\sqrt{\Gamma_{L} \Gamma_{R}}=1$ and $q=\sqrt{\Gamma_{R} / \Gamma_{L}}$. The Hecke algebra quotient $(P, M) H_{L-1}(q)$ according to the realization as a Perk-Shultz quantum chain eq. (27) [24, 26] is also indicated.

From table 5 and Theorem 3 we see that the simple diffusion model $\mathfrak{A}$ has, up to degeneracies, the same spectrum as the XXZ chain used in section 4 to described biased diffusion of a single species of particles $\bullet$. In the same way, the spectrum of model $\mathfrak{E}$ is, up to degeneracies, the same as the one found for pair-annihilation in section 4 , with $2 \alpha=D_{L}+D_{R}$. 


\begin{tabular}{|c|ccc|c|}
\hline model & \multicolumn{3}{|c|}{ reactions } & quotient \\
\hline $\mathfrak{A}$ & $A_{n} \circ \leftrightarrow \circ A_{n}$ & & $(2,0)$ \\
$\mathfrak{B}$ & $A_{n} \circ \leftrightarrow \circ A_{n}$ & $A_{n} A_{m} \leftrightarrow A_{m} A_{n}$ & & $(N, 0)$ \\
$\mathfrak{C}$ & $A_{n} \circ \leftrightarrow \circ A_{n}$ & $A_{n} A_{m} \leftrightarrow A_{m} A_{n}$ & $A_{1} A_{1} \rightarrow \circ A_{1}$ & $(N-1,1)$ \\
$\mathfrak{D}$ & $A_{n} \circ \leftrightarrow \circ A_{n}$ & $A_{n} A_{m} \leftrightarrow A_{m} A_{n}$ & $A_{r} A_{r} \rightarrow A_{r \pm 1} A_{r}$ & $(N-2,2)$ \\
$\mathfrak{E}$ & $A_{n} \circ \leftrightarrow \circ A_{n}$ & $A_{r} A_{s} \rightarrow \circ A_{r+s}$ & & $(1,1)$ \\
$\mathfrak{F}$ & $A_{n} \circ \leftrightarrow \circ A_{n}$ & $A_{r} A_{s} \rightarrow \circ A_{r+s}$ & $A_{n} A_{n} \rightarrow A_{n \pm 1} A_{n \pm 1}$ & $(2,1)$ \\
\hline
\end{tabular}

Table 5: Some integrable reaction-diffusion processes of $N-1$ species and their Hecke algebra quotient [26], see text for the definition of the rates.

For illustration, we briefly consider model $\mathfrak{E}$ for $N=3$. Each site may contain either a particle of type $A$ or $B$ or be empty (o). Single particles may diffuse to the right $A \circ \rightarrow \circ A, B \circ \rightarrow \circ B$ with a rate $\Gamma_{R}$ or similarly to the left with rate $\Gamma_{L}$. On encounter, between like particles the reactions $A A \rightarrow \circ B$ and $A A \rightarrow B \circ$ occur with rates $\Gamma_{R}$ and $\Gamma_{L}$ respectively and similarly $B B \rightarrow \circ A, A \circ$. Two unlike particles react $A B \rightarrow \circ$ with rate $\Gamma_{L}+\Gamma_{R}$. In the left-right symmetric case, the identity of the spectra of $H_{(\mathfrak{E})}$ and the one of pair-annihilation, up to degeneracies, was checked directly [31]. Furthermore, in the spirit of the empty-interval method, a closed system of equations of motion was found, whose solution leads to the mean particledensities $\bar{n}_{A}(t) \sim \bar{n}_{B}(t) \sim t^{-1 / 2}$ [63].

In 64, Bethe-ansatz solutions of the master equation for $N$-species models with particle-numbers conservation are studied. In particular, model $\mathfrak{B}$ with $N=3,5$ was rediscovered. The models in 64 are found from solutions of quantum Yang-Baxter equations. Further study might reveal a relationship to diffusion algebras [39], see below.

For periodic boundary conditions and $N>2$, the diffusion bias leads after a similarity transformation to a generalized Dzialoshinsky-Moriya interaction 65]. A sufficient criterion for integrability was derived in an attempt to look more systematically for integrable many-species models 66.

Finally, a different generalization from section 4 is to consider integrable stochastic models on ladders [71, rather than chains.

F) Diffusion algebras. For certain integrable systems, there exist algebraic methods which allow to find the steady-state $|s\rangle$ such as the celebrated matrix product states [2, 67]. Time-dependent problems are treated in [68]. 
Behind this seemingly technical and ad hoc method there is a new and general mathematical structure. We shall explain here the main idea using reaction-diffusion systems with $N$ states per site labelled by $A_{n}, n=$ $0,1, \ldots, N-1$ (where $A_{0}=0$ ) moving on a periodic chain with $L$ sites, but generalizations to different boundary conditions are possible. The allowed reactions are $A_{n} A_{m} \rightarrow A_{m} A_{n}$ with rate $g_{n m}$ (in particular, model $\mathfrak{B}$ from table 5 is a special case of this). The un-normalized steady-state probability distribution is 2

$$
P_{s}(\boldsymbol{\sigma})=P\left(\sigma_{1}, \ldots, \sigma_{L}\right)=\operatorname{Tr}\left(\mathcal{D}_{\sigma_{1}} \mathcal{D}_{\sigma_{2}} \cdots \mathcal{D}_{\sigma_{L}}\right)
$$

where the matrices $\mathcal{D}_{\sigma}$ satisfy the quadratic relations

$$
g_{\sigma \rho} \mathcal{D}_{\sigma} \mathcal{D}_{\rho}-g_{\rho \sigma} \mathcal{D}_{\rho} \mathcal{D}_{\sigma}=x_{\rho} \mathcal{D}_{\sigma}-x_{\sigma} \mathcal{D}_{\rho}
$$

where $\sigma<\rho$ and $\sigma, \rho \in\{1, \ldots, N\}$ and $g_{\sigma \rho} \in \mathbb{R} \backslash\{0\}, g_{\rho \sigma} \in \mathbb{R}$ and the $x_{\sigma}$ are complex parameters. If in addition the set $\mathcal{A}$ of generators $\mathcal{D}_{\sigma}$ admits a linear PBW basis of ordered monomials $\mathcal{D}_{\sigma_{1}}^{k_{1}} \mathcal{D}_{\sigma_{2}}^{k_{2}} \cdots \mathcal{D}_{\sigma_{n}}^{k_{n}}$ with $\sigma_{1}>\sigma_{2}>\ldots>\sigma_{n}$ and $k_{n} \in \mathbb{N}, \mathcal{A}$ is called a diffusion algebra [39].

These conditions imply certain constraints on the $g_{\sigma \rho}$ and the $x_{\sigma}$, quite analogously to the Jacobi identities of a Lie algebra. These constraints can be fully solved and a classification of all diffusion algebras for $N$ species is obtained [39, 69]. The representation theory of $N$-species diffusion algebras is just getting started, see [70].

\section{Outlook: local scale invariance}

We finish with a discussion on how the scale invariance of many reactiondiffusion systems might be turned into a dynamical symmetry. For example, the symmetric pair-annihilation process is on the Pokrovsky-Talapov critical line. One has the covariance

$$
<n\left(r_{1}, t_{1}\right) \ldots n\left(r_{p}, t_{p}\right)>=b^{-\left(x_{1}+\cdots+x_{p}\right)}<n\left(r_{1}^{\prime}, t_{1}^{\prime}\right) \ldots n\left(r_{p}^{\prime}, t_{p}^{\prime}\right)>
$$

of the $p$-point correlators under the dilatation $r \rightarrow r^{\prime}=b r, t \rightarrow t^{\prime}=b^{z} t$ of the space and time coordinates $r, t$ respectively, where $z$ is the dynamical exponent and $x_{1}, \ldots x_{p}$ are scaling dimensions. In the cases at hand, $z=2$.

This is reminiscent of the situation at equilibrium critical points. In those systems, it is known that under fairly general conditions, the covariance of the $p$-point correlators under global scale transformations $\boldsymbol{r} \rightarrow b \boldsymbol{r}$ can 

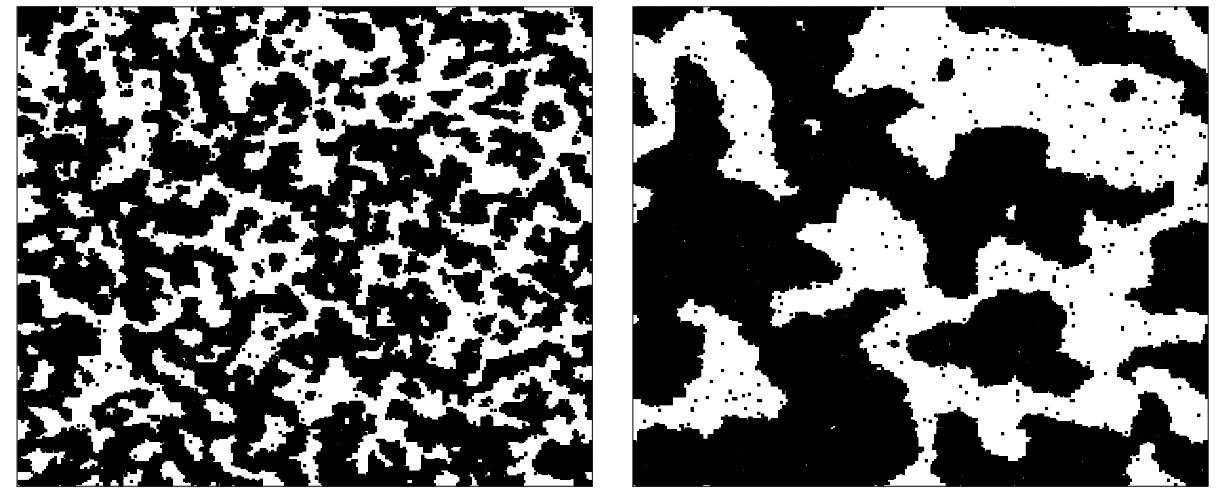

Figure 4: Snapshot of the coarsening of ordered domains in the $2 D$ GlauberIsing model, after a quench to $T=1.5<T_{c}$ from a totally disordered state and at times $t=25$ (left) and $t=275$ (right) after the quench.

be extended to conformal transformations. In addition, in two dimensions the energy-momentum tensor of local conformally invariant field theories becomes an analytic function $T=T(\mathfrak{z})$ of the complex coordinate $\mathfrak{z}$ such that not only $T(\mathfrak{z})$ itself, but also all powers $T^{n}(\mathfrak{z}), n=1,2,3 \ldots$, are conserved [72, 73]. This signals the integrability of $2 D$ conformally invariant field theories. Is it possible to generalize the space-time dilatations encountered for critical reaction-diffusion systems in a similar way?

This question has been recently addressed in the context of kinetic spinsystems [74, 75]. We have already seen above that the kinetic Ising model with Glauber dynamics [20] may be obtained through a similarity transformation of the quantum Hamiltonian from a certain single-species reactiondiffusion system, see model G from table 4 . We now concentrate on this system. In the Glauber-Ising model the transition rates in the master equation are chosen such that the steady-state $|s\rangle$ is given by the equilibrium probability distribution $P_{s}(\boldsymbol{\sigma}) \sim e^{-\mathcal{H}[\boldsymbol{\sigma}] / T}$ with the classical Ising model Hamiltonian $\mathcal{H}=-\sum_{(i, j)} \sigma_{i} \sigma_{j}$ where $T$ is the temperature. Glauber dynamics may be realized through the discrete-time heat-bath rule $\sigma_{i}(t) \rightarrow \sigma_{i}(t+1)$ such that

$$
\sigma_{i}(t+1)= \pm 1 \quad \text { with probability } \frac{1}{2}\left[1 \pm \tanh \left(h_{i}(t) / T\right)\right]
$$

with the local field $h_{i}(t)=h+\sum_{j(i)} \sigma_{j}(t)$. With the choice (78), the master equation can be solved exactly in $1 D$ [20]. The time-dependent spin-spin correlators and their approach towards equilibrium are thus determined. 
In contrast to equilibrium statistical mechanics, where fine-tuning the model parameters is needed to reach a critical point, dynamical scaling is often found to occur in large regions of the model's parameter space. For example, prepare the system initially in a disordered state and then quench the temperature to a final temperature $T<T_{c}$ below the critical temperature $T_{c}>0 .{ }^{6}$ Although the steady-state of the model is not critical, the relaxation towards it occurs through domain coarsening and is very slow, the typical length scale varying with time as $L(t) \sim t^{1 / z}$, see figure 4 . Typically the observables depend algebraically on the time $t$ passed since the quench, see [8, 9] for (a collection of) recent reviews. Here we concentrate on the twotime spatio-temporal response function $R(t, s ; \boldsymbol{r})$ of the time-dependent spin $\sigma_{\boldsymbol{r}}(t)$ at site $\boldsymbol{r}$ with respect to an external magnetic field $h_{\mathbf{0}}(s)$ applied at the origin $\mathbf{0}$ at an earlier time $s<t$. Generically, two-time quantities such as $R(t, s ; \boldsymbol{r})$ depend on both times $t$ and $s$ and not merely on the difference $\tau=t-s$. This breaking of time-translation invariance is called ageing.

For ageing systems, an extension of dynamical scaling is possible and allows to fix the form of the two-time response function. Specifically, it can be shown that for a dynamical exponent $z=2$ [77, 74, 75]

$R(t, s ; \boldsymbol{r})=\left.\frac{\delta\left\langle\sigma_{\boldsymbol{r}}(t)\right\rangle}{\delta h_{\mathbf{0}}(s)}\right|_{h=0}=r_{0}\left(\frac{t}{s}\right)^{1+a-\lambda_{R} / 2}(t-s)^{-1-a} \exp \left[-\frac{\mathcal{M}}{2} \frac{\boldsymbol{r}^{2}}{t-s}\right]$

Here $a$ and $\lambda_{R}$ are non-equilibrium exponents to be determined which characterize the ageing universality class [78. Finally, $r_{0}$ and $\mathcal{M}$ are nonuniversal constants. We first present evidence that the response function of the Glauber-Ising model in $2 D$ and $3 D$ is indeed given by (79). Then we discuss where this presumably exact result comes from.

We first consider the autoresponse $R(t, s)=R(t, s ; \mathbf{0})$. While $R$ itself is too noisy to be measured directly, integrated response functions are accessible through simulations, see [79] and references therein for the details which we skip over here. For the example, the integrated autoresponse

$$
\rho(t, s)=\int_{0}^{s} \mathrm{~d} u R(t, u) \sim s^{-a} f_{M}(t / s)
$$

is relatively easy to measure, whereas the scaling function $f_{M}(x)$ can be calculated explicitly from (79). In the Glauber-Ising model, the exponent

\footnotetext{
${ }^{6}$ In the $1 D$ Glauber-Ising model, $T_{c}=0$ leads to certain modifications of the ageing as described from the point of view of local scale invariance [76].
} 

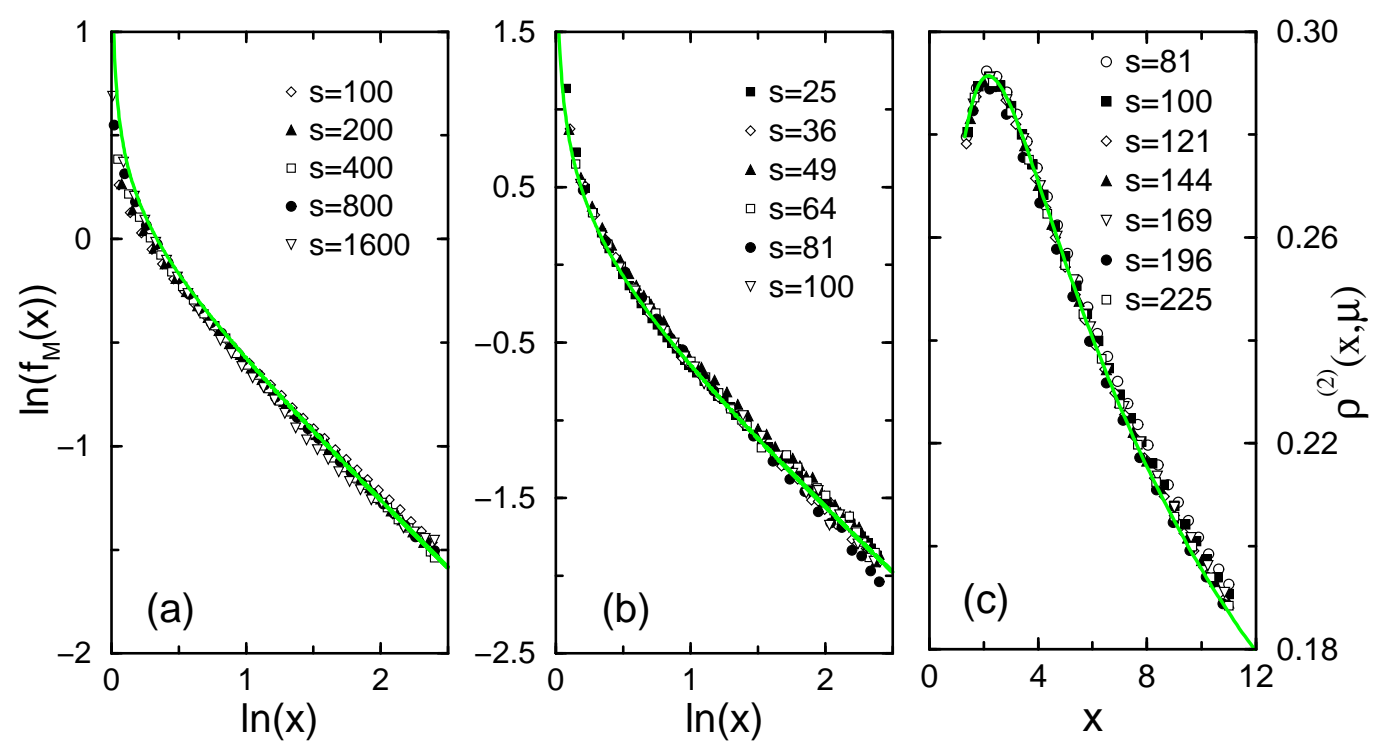

Figure 5: Scaling form of the integrated magnetic response in the GlauberIsing model as a function of $x=t / s$ below criticality. The symbols correspond to different waiting times $s$. The integrated autoresponse is shown (a) $2 D$ at $T=1.5$ and (b) $3 D$ at $T=3$. An example of the integrated spatio-temporal response in $2 D$ at $T=1.5$ and with $\mu=2$ is shown in (c). The full curves are obtained from (79) $)$. After [79].

$a=1 / z=1 / 2$ (see [80] for a detailed discussion) and $\lambda_{R} \simeq 1.26$ and 1.6 in $2 D$ and $3 D$, respectively. In figure $5 \mathrm{ab}$, the scaling function $f_{M}(x)$, as obtained from large-scale simulations, is shown for several values of the waiting time $s$. In both two and three dimensions, a nice scaling behaviour is found and the form of the scaling function agrees very well with the prediction from eq. (79) . Next, the $\boldsymbol{r}$-dependence of $R(t, s ; \boldsymbol{r})$ is tested by measuring the spatio-temporally integrated response $\int_{0}^{s} \mathrm{~d} u \int_{0}^{\sqrt{\mu s}} \mathrm{~d} r r^{d-1} R(t, u ; \boldsymbol{r}) \sim$ $s^{d / 2-a} \rho^{(2)}(t / s, \mu)$, where $\mu$ is a control parameter. We stress that the scaling function $\rho^{(2)}$ does not contain any more free non-universal parameter [79]. As an example, we compare in figure 5: data from $2 D$ taken with $\mu=2$ with eq. (79). Besides the expected scaling, the functional form of the scaling function neatly follows the prediction. We stress that the position, the height and the width of the maximum of $\rho^{(2)}$ in figure 5t are completely fixed. Similar results have been obtained for other values of $\mu$ and in $3 D$ as well. 
This provides strong evidence that eq. (79) is exact, at least in this model [79. Tests of (79) in different universality classes are described in [75].

In order to derive (79), consider the diffusion equation

$$
\left(2 \mathcal{M} \partial_{t}-\boldsymbol{\partial}_{\boldsymbol{r}} \cdot \boldsymbol{\partial}_{\boldsymbol{r}}\right) \phi(t, \boldsymbol{r})=0
$$

For fixed $\mathcal{M}$, the Schrödinger group is the maximal invariance group on the space of solutions of eq. (81). It is defined by the space-time transformations $(\mathcal{R}$ is a rotation matrix)

$$
t \longmapsto t^{\prime}=\frac{\alpha t+\beta}{\gamma t+\delta}, \boldsymbol{r} \longmapsto \boldsymbol{r}^{\prime}=\frac{\mathcal{R} \boldsymbol{r}+\boldsymbol{v} t+\boldsymbol{a}}{\gamma t+\delta} ; \alpha \delta-\beta \gamma=1
$$

and acts projectively on the solutions $\phi(t, \boldsymbol{r})$ [81. Let $\mathfrak{s c h} \mathfrak{h}_{d}$ be the Lie algebra of (82). Time-translations occur in $\mathfrak{s c h}_{d}$ and are parametrized by $\beta$. If we treat the 'mass' $\mathcal{M}$ not as a constant but as another variable, the embedding $\mathfrak{s c h}_{d} \subset \mathfrak{c o n f}_{d+2}$ for the complexified Lie algebras follows [82, where $\mathfrak{c o n f}_{d+2}$ is the Lie algebra of the conformal group in $d+2$ dimensions. From the classification of the parabolic subalgebras of $\mathfrak{c o n f}_{d+2}$ we obtain several new subalgebras, called $\widetilde{\mathfrak{a g e}}$ or $\widetilde{\mathfrak{a l t}}[82$. For the $1 D$ case, we illustrate in figure 6 their definition through the root space of $\mathfrak{c o n f}_{3} \cong B_{2}$. These subalgebras still contain the generator for the dilatations $t \rightarrow b^{2} t, \boldsymbol{r} \rightarrow b \boldsymbol{r}$ (which is in the Cartan subalgebra of $\mathfrak{c o n f}_{3}$ ) but do not contain time-translations anymore (which is in the lower left corner of figure 6abc). They are candidates for a dynamic symmetry algebra of ageing systems. If we assume that the two-time response function transforms covariantly under the action of either $\widetilde{\mathfrak{a g} \mathfrak{e}}$ or $\widetilde{\mathfrak{a l t}}$, a set of linear differential equations for $R(t, s ; \boldsymbol{r})$ is obtained. Matching their solution with the expected [78] scaling behaviour of $R$, we recover eq. (779) in the special case $z=2$.

The functional form of $R$ depends on the fact that the Galilei transformation of (82) is identical to the well-known one of a free particle. It is not trivial at all that the response function of an interacting field theory such as the Glauber-Ising model in $d>1$ dimensions should be recovered from a dynamical symmetry of the equation of motion of a free-field theory.

There exist infinite-dimensional Lie algebras which contain $\mathfrak{s c h}_{d}$ as subalgebras. For example, the Schrödinger group (82) is a subgroup of the group defined by the transformations $t \rightarrow t^{\prime}$ and $\boldsymbol{r} \rightarrow \boldsymbol{r}^{\prime}$ where

$$
t^{\prime}=\beta(t), \quad \boldsymbol{r}^{\prime}=\boldsymbol{r} \sqrt{\dot{\beta}(t)} \quad \text { or else } \quad t^{\prime}=t, \quad \boldsymbol{r}^{\prime}=\boldsymbol{r}-\boldsymbol{\alpha}(t)
$$




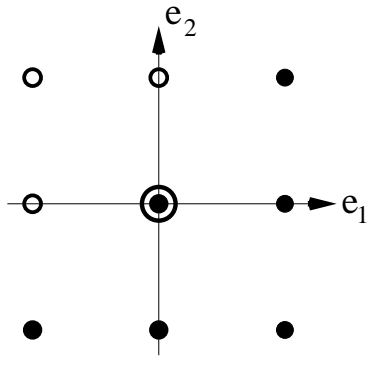

( a )

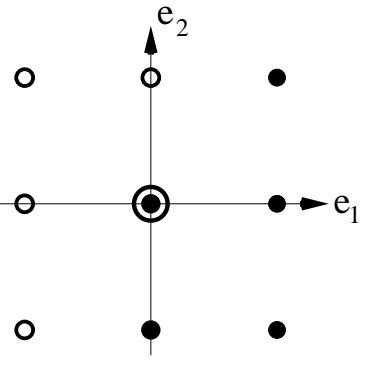

( b )

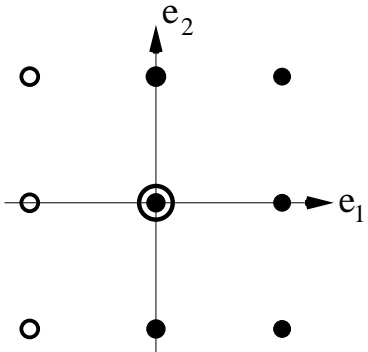

(c)

Figure 6: Root space of the complexified conformal Lie algebra $\mathfrak{c o n f}_{3}$, indicated by the full and the open points. The double circle in the center denotes the Cartan subalgebra. The generators belonging to the three nonisomorphic parabolic subalgebras 82 are indicated by the full points, namely (a) $\widetilde{\mathfrak{s c h}}_{1}$, (b) $\widetilde{\mathfrak{a} \mathfrak{g} \mathfrak{e}_{1}}$ and (c) $\widetilde{\mathfrak{a l t}}_{1}$.

and $\beta$ and $\boldsymbol{\alpha}$ are arbitrary functions. Whether this has a bearing on the ageing behaviour of non-equilibrium spin-systems is still open. Local scale transformations generalizing the Schrödinger group (82) to general values of the dynamical exponent $z \neq 2$ exist $[75$. It can be shown that $R(t, s ; \boldsymbol{r})=$ $R(t, s ; \mathbf{0}) \Phi\left(r(t-s)^{-1 / z}\right)$, such that eq. (79) holds for the autoresponse $R(t, s ; \mathbf{0})$ if $\lambda_{R} / 2$ is replaced by $\lambda_{R} / z$ and $\Phi(v)$ is given as the solution of a linear differential equation of fractional order [75].

I thank M. Pleimling and J. Unterberger for their pleasant collaboration on local scale invariance. This work was supported by CINES Montpellier (projet pmn2095) and the Bayerisch-Französisches Hochschulzentrum (BFHZ).

\section{References}

[1] B. Derrida, J. L. Lebowitz, and E. R. Speer, Phys. Rev. Lett. 89, 030601 (2002).

[2] B. Derrida, S.A. Janowsky, J.L. Lebowitz and E.R. Speer, J. Stat. Phys. 73, 813 (1993); B. Derrida, Phys. Rep. 301, 65 (1998).

[3] B. Schmittmann and R.K.P. Zia, in: Phase Transitions and Critical Phenomena (C. Domb and J. Lebowitz Eds.), Vol. 17, Academic Press (London, 1995). 
[4] V. Privman (ed.) Nonequlibrium Statistical Mechanics in One Dimension, Cambridge University Press (Cambridge 1996).

[5] J. Marro and R. Dickman, Nonequilibrium phase transitions in lattice models, Cambridge University Press (Cambridge, 1999).

[6] H. Hinrichsen, Adv. Phys. 49, 815 (2000).

[7] D. ben-Avraham and S. Havlin, Diffusion and reactions in fractals and disordered systems, Cambridge University Press (Cambridge 2000).

[8] M.E. Cates and M.R. Evans (eds), Soft and Fragile Matter, IOP Press (Bristol 2000).

[9] L.F. Cugliandolo, cond-mat/0210312

[10] G.M. Schütz, in: Phase Transitions and Critical Phenomena (C. Domb and J. Lebowitz Eds.), Vol. 19, Academic Press (London 2000).

[11] M.v. Smoluchowski, Z. Phys. Chem. 92, 129 (1917).

[12] D. ben-Avraham, M.A. Burschka and C.R. Doering, J. Stat. Phys. 60, 695 (1990).

[13] J.L. Spouge, Phys. Rev. Lett. 60, 871 (1988); err. 60, 1885 (1988).

[14] D. Toussaint and F. Wilczek, J. Chem. Phys. 78, 2642 (1983).

[15] J. Prasad and R. Kopelman, Chem. Phys. Lett. 157, 535 (1989).

[16] R. Kopelman, C. S. Li and Z.-Y. Shi, J. Luminescence 45, 40 (1990).

[17] R. Kroon, H. Fleurent and R. Sprik, Phys. Rev. E47, 2462 (1993).

[18] S. Cornell and M. Droz, Phys. Rev. Lett. 70, 3824 (1993).

[19] for a forthcoming review, see M. Henkel and H. Hinrichsen, (2003)

[20] R. J. Glauber, J. Math. Phys. 4, 294 (1963).

[21] C. Hyver, J. Theor. Biol. 36, 133 (1972); J. Keizer, J. Stat. Phys. 6, 67 (1972); J. Schnakenberg, Rev. Mod. Phys. 48, 571 (1976).

[22] P.P. Martin, Potts model and related problems in statistical mechanics, World Scientific (Singapour 1991). 
[23] P.P. Martin and V. Rittenberg, Int. J. Mod. Phys. A7 Suppl. 1B, 707 (1992) and B4, 792 (1992).

[24] C.L. Schultz, Phys. Rev. Lett. 46, 629 (1981); Physica 122, 71 (1983); J.H.H. Perk and C.L. Schultz, in: Non-Linear Integrable Systems, Classical Theory and Quantum Theory, (M. Jimbo and T. Miwa Eds.), World Scientific (Singapour 1981).

[25] V.R. Jones, Int. J. Mod. Phys. B4, 701 (1990).

[26] F.C. Alcaraz and V. Rittenberg, Phys. Lett. B314, 377 (1993).

[27] A.N. Kirrilov and N. Yu. Reshetikhin, LOMI preprint (1988).

[28] V. Pasquier and H. Saleur, Nucl. Phys. B330, 523 (1990).

[29] H. Saleur, in Proc. of Trieste Conf. on Recent Developments in Conformal Field Theories (1989).

[30] S. Alexander and T. Holstein, Phys. Rev. B18, 301 (1978).

[31] F.C. Alcaraz, M. Droz, M. Henkel and V. Rittenberg, Ann. of Phys. 230, 250 (1994).

[32] D. Kandel, E. Domany and B. Nienhuis, J. Phys. A23, L557 (1990).

[33] G. Schütz, J. Stat. Phys. 71, 471 (1993).

[34] F. C. Alcaraz, D. Arnaudon, V. Rittenberg and M. Scheunert, Int. J. Mod. Phys. A9, 3473 (1994).

[35] G.M. Schütz, J. Stat. Phys. 79, 243 (1995); A. Aghamohammadi and M. Khorrami, J. Phys. A34, 7431 (2001).

[36] S. Albeverio and S.-M. Fei, Rev. Math. Phys. 10, 723 (1998)

[37] F.C. Alcaraz, S. Dasmahapatra and V. Rittenberg, J. Phys. A31, 845 (1998).

[38] S. Grosskinsky, G.M. Schütz and H. Spohn, cond-mat/0302079.

[39] A.P. Isaev, P.N. Pyatov and V. Rittenberg, J. Phys. A34, 5815 (2001).

[40] M. Henkel, E. Orlandini and J. Santos, Ann. of Phys. 259, 163 (1997).

[41] I. Peschel, V. Rittenberg and U. Schultze, Nucl. Phys. B430, 633 (1994). 
[42] L.-H. Gwa and H. Spohn, Phys. Rev. A46, 844 (1992); D. Kim, Phys. Rev. E52, 3512 (1995).

[43] S. Sandow and G. Schütz, Europhys. Lett. 26, 7 (1994).

[44] J.D. Johnson and B.M. McCoy, Phys. Rev. A6, 1613 (1972); M. Takanishi, Prog. Theor. Phys. 50, 1519 (1973).

[45] M. Henkel and G. M. Schütz, Physica A206, 187 (1994).

[46] J. Krug, Phys. Rev. Lett. 67, 1882 (1991).

[47] F.C. Alcaraz, M.N. Barber and M.T. Batchelor, Ann. of Phys. 182, 280 (1988).

[48] F.C. Alcaraz, M.N. Barber, M.T. Batchelor, R.J. Baxter and G.R.W. Quispel, J. Phys. A20, 6397 (1987).

[49] R.J. Baxter, J. Stat. Phys. 108, 1 (2002).

[50] R.J. Baxter, Exactly solved models in statistical mechanics, Academic Press (London 1982).

[51] M. Gaudin, La fonction d'onde de Bethe, Masson (Paris 1983).

[52] K. Krebs, M.P. Pfannmüller, B. Wehefritz and H. Hinrichsen, J. Stat. Phys. 78, 1429 (1995).

[53] M. Henkel, E. Orlandini and G. M. Schütz, J. Phys. A28 (1995), 6335.

[54] H. Simon, J. Phys. A28, 6585 (1995).

[55] A.A. Lushnikov, Sov. Phys. JETP 64, 811 (1986); Phys. Lett. 120A, 135 (1987).

[56] E. Siggia, Phys. Rev. B16, 2319 (1977).

[57] J.E. Santos, J. Phys. A30, 3249 (1997).

[58] A. Aghamohammadi and M. Khorrami, J. Phys. A33, 7843 (2000).

[59] M. Henkel and H. Hinrichsen, J. Phys. A34, 1561 (2001).

[60] M. Khorrami, A. Aghamohammadi and M. Alimohammadi, J. Phys. A36, 345 (2003).

[61] T. Masser and D. ben-Avraham, Phys. Rev. E63, 066108 (2001). 
[62] T. Masser and D. ben-Avraham, cond-mat/0106306.

[63] V. Privman, Phys. Rev. A46, R6140 (1992).

[64] M. Alimohammadi and N. Ahmadi, J. Phys. A35, 1325 (2002).

[65] S. R. Dahmen, J. Phys. A28, 905 (1995).

[66] V. Popkov, M.E. Fouladvand and G.M. Schütz, J. Phys. A35, 7187 (2002).

[67] B. Derrida, M.R. Evans, V. Hakim and V. Pasquier, J. Phys. A26, 1493 (1993).

[68] V. Popkov and G.M. Schütz, Matem. Fisika Anal. Geom., 9, 401 (2002);

R.B. Stinchcombe and G.M. Schütz, Phys. Rev. Lett. 75, 140 (1995).

[69] P.N. Pyatov and R. Twarock, J. Math. Phys. 43, 3268 (2002).

[70] R. Twarock, in: Proc. on Quantum Theory and Symmetries, (E. Kapuscik and A. Horzela Eds.), World Scientific (Singapour 2002), p. 615.

[71] S. Albeverio and S.-M. Fei, J. Phys. A34, 6545 (2001).

[72] A.A. Belavin, A.M. Polyakov and A.B. Zamolodchikov, Nucl. Phys. B241, 333 (1984).

[73] A.B. Zamolodchikov, Adv. Stud. Pure Math. 19, 641 (1989).

[74] M. Henkel, M. Pleimling, C. Godrèche and J.-M. Luck, Phys. Rev. Lett. 87, $265701(2001)$

[75] M. Henkel, Nucl. Phys. B641, 405 (2002).

[76] A. Picone and M. Henkel, in preparation.

[77] M. Henkel, J. Stat. Phys. 75, 1023 (1994).

[78] C. Godrèche and J.-M. Luck, J. Phys. Cond. Matt. 14, 1589 (2002).

[79] M. Henkel and M. Pleimling, cond-mat/0302482.

[80] M. Henkel, M. Paessens and M. Pleimling, Europhys. Lett. in press (cond-mat/0211583).

[81] U. Niederer, Helv. Phys. Acta 45, 802 (1972).

[82] M. Henkel and J. Unterberger, Nucl Phys. B in press (hep-th/0302187).

[83] A. Picone and M. Henkel, J. Phys. A35, 5575 (2002). 